\title{
TSG-6 attenuates inflammation-induced brain injury via modulation of microglial polarization in SAH rats through the SOCS3/STAT3 pathway
}

Ran Li, Wenchao Liu, Jian Yin, Yunchang Chen, Shenquan Guo, Haiyan Fan, Xifeng Li, Xin Zhang, Xuying He and Chuanzhi Duan*

\begin{abstract}
Background: An acute and drastic inflammatory response characterized by the production of inflammatory mediators is followed by stroke, including SAH. Overactivation of microglia parallels an excessive inflammatory response and worsened brain damage. Previous studies indicate that TSG-6 has potent immunomodulatory and anti-inflammatory properties. This study aimed to evaluate the effects of TSG-6 in modulating immune reaction and microglial phenotype shift after experimental SAH.

Methods: The SAH model was established by endovascular puncture method for Sprague-Dawley rats (weighing 280-320 g). Recombinant human protein and specific siRNAs for TSG-6 were exploited in vivo. Brain injury was assessed by neurologic scores, brain water content, and Fluoro-Jade C (FJC) staining. Microglia phenotypic status was evaluated and determined by Western immunoblotting, quantitative real-time polymerase chain reaction (qPCR) analyses, flow cytometry, and immunofluorescence labeling.

Results: SAH induced significant inflammation, and M1-dominated microglia polarization increased expression of TSG-6 and neurological dysfunction in rats. rh-TSG-6 significantly ameliorated brain injury, decreased proinflammatory mediators, and skewed microglia towards a more anti-inflammatory property 24-h after SAH. While knockdown of TSG6 further induced detrimental effects of microglia accompanied with more neurological deficits, the anti-inflammation effects of rh-TSG-6 were associated with microglia phenotypic shift by regulating the level of SOCS3/STAT3 axis.

Conclusions: TSG-6 exerted neuroprotection against SAH-induced EBI in rats, mediated in part by skewing the balance of microglial response towards a protective phenotype, thereby preventing excessive tissue damage and improving functional outcomes. Our findings revealed the role of TSG-6 in modulating microglial response partially involved in the SOCS3/STAT3 pathway and TSG-6 may be a promising therapeutic target for the treatment of brain injury following SAH.
\end{abstract}

Keywords: Subarachnoid hemorrhage, Early brain injury, Microglial polarization, TSG-6, Anti-inflammation

\footnotetext{
* Correspondence: doctor_duanzj@163.com

Department of Neurosurgery, Zhujiang Hospital, The National Key Clinical Specialty, The Neurosurgery Institute of Guangdong Province, Guangdong Provincial Key Laboratory on Brain Function Repair and Regeneration,

Southern Medical University, Guangzhou 510282, China
}

(c) The Author(s). 2018 Open Access This article is distributed under the terms of the Creative Commons Attribution 4.0 International License (http://creativecommons.org/licenses/by/4.0/), which permits unrestricted use, distribution, and reproduction in any medium, provided you give appropriate credit to the original author(s) and the source, provide a link to the Creative Commons license, and indicate if changes were made. The Creative Commons Public Domain Dedication waiver (http://creativecommons.org/publicdomain/zero/1.0/) applies to the data made available in this article, unless otherwise stated. 


\section{Background}

Subarachnoid hemorrhage (SAH) constitutes 5 to $10 \%$ of all strokes worldwide [1]. Healthy people can harbor intracranial aneurysms noted in occasional examination or emerging with several symptoms, otherwise detected with SAH onset [2, 3]. Once intracranial aneurysm ruptures, SAH is usually catastrophic because there exists no effective therapy applied in concomitant brain injury [4]. Currently, early brain injury (EBI) and delayed cerebral ischemia (DCI) represent the main effects of SAH at two stages, and neuroprotection and anti-vasospasm are the most studied targets in numerous research $[5,6]$. Though many putative agents demonstrate exciting therapeutic effects, little has been translated into clinical application, and many have failed clinical trials [7]. Only nimodipine has been widely used in clinical application and has proven effective in the treatment of SAH [8].

$\mathrm{SAH}$ can induce several external stress events, including rapid ascension of intracranial pressure, sharp reduction in cerebral perfusion pressure, brain edema, and heme burden from red blood cell lysis, all of which converged to result in cellular pathophysiological changes [9]. Several studies have indicated that inflammation is invariably associated with brain damage after SAH [1012]. As shown in clinical data, a sign of early inflammation in aSAH patients is correlated with poor neurological outcome on admission [13]. Microglia may sense even small imbalances of environmental homeostasis and are rapidly activated in a mode of dynamic morphology and polarization [14]. Afterwards, activated microglia appear to be the predominant source for a plethora of inflammatory mediators in the central nervous system (CNS) [15]. The progression of a dysfunctional and highly reactive microglial activation results in releasing high levels of pro-inflammatory and cytotoxic mediators that contribute to neuronal dysfunction and cell death [16]. The secreted cytokines together with environmental toxins and endogenous proteins, combined with neuronal death, further provoke unregulated activation of microglia resulting in the production of toxic factors that can propagate inflammation-induced neuronal death [17]. Therefore, alternative strategies based on a clearer comprehension of microglia-mediated inflammation are pressingly warranted. Accordingly, microglia, the prominent responder during neuroinflammtion, has been identified as the target in the present study.

Microglia polarization in different phenotypes exerting distinct responses is a central feature of disease progression involved inflammation [18]. Activated microglia are assumed to polarize into two extreme states: classical (M1, proinflammatory) and alternative (M2, anti-inflammatory) activation. Microglia dynamically transfer between M1 and M2 phenotypes after activation. Meanwhile, each phenotype can be identified by typical biomarkers. M1 phenotypic cells are characterized by the expression of CD86, CD68, iNOS, etc. Likewise, M2 phenotypic cells are associated with the expression of CD163, CD206, arginase-1(Arg-1), etc. In addition, M1 microglia are associated with a proinflammatory cellular state that has elevated expression of inflammatory cytokines, including IL- $1 \beta$, TNF- $\alpha$, and IL-6, which enhance brain damage. In contrast, M2 microglia release anti-inflammatory mediators, including IL-4, IL-10, and TGF- $\beta$, leading to beneficial neuroprotection $[19,20]$. Though this M1/M2 paradigm is an oversimplified schema that simply divides the activated microglia populations into M1 and M2 phenotypes, it remains the most commonly used model to understand the role of microglia.

The phenomena of polarization of microglia has been carefully confirmed in several CNS diseases model. Fine-tuned microglia M1/M2 polarization has obtained positive therapeutic efficacy in experimental spinal cord injury (SCI), intracerebral hemorrhage (ICH), and ischemic brain injury models by suppressing the deleterious effects of inflammation, while boosting neuroprotective potential [21-23]. Largely, cues in the microenvironment and intertwined intracellular signals may determine how they polarize into $\mathrm{M} 1$ phenotype to exacerbate tissue injury or M2 phenotype to promote tissue recovery [24].

Recently, TSG-6 has emerged as a protective regulator against inflammation in cornea injury, peritonitis, autoimmune diabetes, asthma, and other inflammation-associated diseases. TSG-6 is a multifunctional glycoprotein composed of a hyaluronan-binding link domain and a CUB module in a contiguous fashion [25]. Normally, TSG-6 is upregulated in several pathological contexts especially related to inflammation. Collectively, the therapeutic effects of TSG- 6 can be explained by participating in HA crosslinking and/or catalyzing the transfer of I $\alpha \mathrm{I}$ heavy chains to HA [26]. It is, therefore, likely that TSG- 6 acts as an endogenous inhibitor comprising part of a negative feedback loop in inflammation progression. Different from peripheral tissue, microglia play a major immune-response function instead of macrophages in the brain. However, the role of TSG-6 within CNS remains unclear. Our laboratory recently discovered that TSG-6 may preserve blood-brain barrier (BBB) by attenuating nitrative stress in an ICH model and improve outcomes in animal models of TBI by reducing the activation of microglia/macrophages [27, 28]. These results indicate that TSG-6 can play protective roles in CNS. However, whether TSG-6 impacts microglia polarization remains to be reported.

Herein, we aimed to address the possibility of TSG-6 in the regulation of microglia-mediated inflammation and the effects of TSG- 6 on microglial polarization after 
SAH injury, as well as its potential mechanism in a rat endovascular puncture model of SAH.

\section{Methods \\ Animals}

Sprague-Dawley male rats (280-320 g) were obtained from the Animal Experiment Center of Southern Medical University. All experimental procedures and animal care were approved by the Southern Medical University Ethics Committee and were in accordance with the guidelines of the National Institute of Health. All rats resided in a light and temperature-controlled environment with ad libitum access to food and water and adapted to the environment 1 week before the experiments.

\section{Experimental design and animal groups Time course and cell distribution}

In this experiment, 84 male rats were divided into six groups at random (sham and, SAH 6, 12, 24, 48, $72 \mathrm{~h}$ after $\mathrm{SAH})$. The mRNA and protein expression level and time course of TSG-6 were measured by qPCR and Western blot. Expression distribution was detected by FISH-ISH and double immunostaining to determine TSG-6 expression in different cell types of the brain in the sham group and $24 \mathrm{~h}$ after the SAH group. In additional study, qPCR and western blot were also employed to detect whether there was a statistical difference in TSG-6 gene and protein levels among the sham groups at $6 \mathrm{~h}, 12 \mathrm{~h}, 24 \mathrm{~h}, 48 \mathrm{~h}$, and $72 \mathrm{~h}$.

\section{Outcomes of treatment}

To assess the role of TSG-6 on early brain injury after SAH, 102 rats were randomly divided into the sham group, $\mathrm{SAH}$ group, $\mathrm{SAH}+$ vehicle group, $\mathrm{SAH}+$ rh-TSG-6 $(1 \mu \mathrm{g})$, SAH + rh-TSG-6 $(5 \mu \mathrm{g}), \mathrm{SAH}+\mathrm{scram}-$ bled siRNA group, and SAH + TSG-6 siRNA group. All the rats were sacrificed $24 \mathrm{~h}$ after SAH according to the results of the first experiment. siRNA transfection efficiencies of each sample were verified using western blot analysis. Neurological scores, brain water content, and fluoro-Jade C (FJC) analysis were conducted.

\section{Correlation between TSG- 6 and microglia polarization}

To examine the effect of TSG- 6 on microglia polarization, 60 rats were randomly assigned into the following groups: sham, SAH, SAH + vehicle, SAH + rh-TSG-6 $(5 \mu \mathrm{g})$, SAH + scrambled siRNA group, and SAH + TSG-6 siRNA groups. Animals were sacrificed for brain tissue $24 \mathrm{~h}$ after SAH onsets. The samples were collected for qPCR, Flow cytometric analysis, ELISA, and immunofluorescence analysis. To clarify, we used samples partly from the first two for immunofluorescence (IF), qPCR, and WB experiments instead of having a separate cohort of SAH rats.

\section{Therapeutic mechanism of action}

To explore the potential mechanism of TSG- 6 on modulating microglia polarization, 36 rats were randomly assigned into the following groups: sham, $\mathrm{SAH}, \mathrm{SAH}+$ vehicle, $\quad \mathrm{SAH}+$ rh-TSG-6 $\quad(5 \mu \mathrm{g}), \quad \mathrm{SAH}+$ scrambled siRNA, and SAH + TSG-6 siRNA groups. Immunofluorescence and western blotting was performed $24 \mathrm{~h}$ after $\mathrm{SAH}$ induction.

\section{Experimental SAH model}

The SAH model was performed by endovascular puncturing for the induction of SAH as previously described [29]. Briefly, rats were deeply anesthetized by $1 \%$ pentobarbital sodium $(40 \mathrm{mg} / \mathrm{kg}$, i.p.). A sharpened 4-0 nylon suture was inserted rostrally into the left internal carotid artery and perforated the bifurcation of the anterior and middle cerebral arteries until resistance was felt. Next, the suture was immediately withdrawn to allow blood reperfusion in the internal carotid artery, induced to SAH. Sham animals underwent the same procedures without vessel perforation.

\section{SAH grade}

After euthanasia and removal of the brain, the basal brain was photographed immediately and divided into six segments as previously described [30]. Based on the amount of blood clotting, each area was blindly assigned a score from 0 to 3 . All area scores were summed as the total SAH grade $($ maximum SAH grade $=18$ ). Experimental rats with mild SAH whose SAH grades $\leq 7$ were excluded from the study.

\section{Neurological score}

The neurological scores were evaluated $24 \mathrm{~h}$ after SAH using the previously described modified Garcia scoring system [30]. Briefly, the evaluation included six tests scored from 0 to 3 or 1 to 3 and included the following: spontaneous activity, symmetry in the movement of four limbs, forelimbs outstretching, climbing ability, body proprioception, and the response to vibrissae stimulation. Possible scores ranged from 3 to 18 . All the tests were evaluated by an observer who was blind to the treatment conditions. Higher scores represented better neurological function.

\section{Brain water content analysis}

Brains were removed at $24 \mathrm{~h}$ after SAH and were divided into four parts: left hemisphere, right hemisphere, cerebellum, and brainstem. Left and right hemispheres were weighed immediately to obtain the wet weight and were then oven dried at $105{ }^{\circ} \mathrm{C}$ for $24 \mathrm{~h}$ to obtain the dry weight. The percentage of water content was calculated as follows: [(wet weight-dry weight)/wet weight] $\times 100 \%$. 
Intracerebroventricular injection administration

In vivo transfection was performed as described previously [31]. TSG-6 siRNA (Santa Cruz Biotechnology, USA) and control scramble SiRNA (Santa Cruz Biotechnology, USA) transfection was performed with in vivo siRNA transfection reagent (Engreen Biosystem, Beijing, China) according to manufacturer protocols. TSG6-siRNA was dissolved in RNase-free $\mathrm{H}_{2} \mathrm{O}$ at concentrations of $1 \mu \mathrm{g} / 1 \mu \mathrm{l}$; an equivalent concentration of scrambled-sequence siRNA was transfected into the negative control. Next, $5 \mu \mathrm{L}$ TSG-6 siRNA or control siRNA was diluted with $5 \mu \mathrm{L}$ in vivo transfection reagent. Finally, the mixture was injected intracerebroventricularly using a $10 \mu \mathrm{l}$ Hamilton microsyringe (Microliter No. 701; Hamilton Company, Switzerland) under the guidance of a stereotaxic instrument (Stoelting Company, USA) under anesthesia. The SAH model was established $48 \mathrm{~h}$ later. rh-TSG-6 was dissolved in sterile PBS to a final concentration of $1 \mu \mathrm{g} / 10 \mu \mathrm{L}$ or $5 \mu \mathrm{g} / 10 \mu \mathrm{L}$. Then, $1 \mu \mathrm{g}$ or $5 \mu \mathrm{g}$ rh-TSG- 6 was infused into the cerebroventricle using a Hamilton syringe with the guidance of a stereotaxic instrument $1.5 \mathrm{~h}$ after SAH induction. The dosage of rh-TSG-6 was determined based on a previous study. The vehicle group were administered the same volume of sterile PBS or RNase-free $\mathrm{H}_{2} \mathrm{O}$.

\section{Quantitative real-time polymerase chain reaction}

Quantitative real-time polymerase chain reaction (qPCR) was performed and analyzed as previously described [27]. Total RNA from brain tissues with blood clots was extracted using TRIzol (Invitrogen, USA). Total RNA was reverse-transcribed to cDNA using the PrimeScript ${ }^{\text {TM }}$ RT reagent Kit with gDNA Eraser (Takara, China). Rt-PCR reactions were performed on the Illumina-Eco Real-Time PCR Detection System (Gene Company Limited, USA) using the SYBR Premix Ex TaqII kit (Takara, China). The running procedure was $30 \mathrm{~s}$ at $95^{\circ} \mathrm{C}, 40 \mathrm{cy}-$ cles of $5 \mathrm{~s}$ at $95{ }^{\circ} \mathrm{C}$, and $30 \mathrm{~s}$ at $60{ }^{\circ} \mathrm{C}$, following a melt curve. Gene expression was quantified with standard samples and normalized with $\beta$-actin. Data were expressed as normalized messenger RNA (mRNA) expression (fold mRNA increase). The real-time PCR primer sequences are listed in Table 1.

\section{Western blotting}

The cerebral cortex tissues with blood clots were collected at corresponding time-points after SAH. Western blot (WB) was performed as described previously [27]. The following primary antibodies were used for WB: mouse anti-TSG-6 (Santa Cruz Biotechnology; 1:800), rabbit anti-STAT3 (Cell Signaling Technology; 1:2000), rabbit anti-phosphorylated STAT3 at Tyr705 (Cell Signaling Technology; 1:1000), rabbit anti-SOCS3 (Abcam;
Table 1 Real-time PCR primers used in this study

\begin{tabular}{|c|c|c|}
\hline Primer name & Primer sequence & \\
\hline \multirow[t]{2}{*}{ TSG-6 } & Forward & CGTCTTGCAACCTACAAGCAGCTA \\
\hline & Reverse & ACAGTTGGGCCCAGGTTTCA \\
\hline \multirow[t]{2}{*}{ CD86 } & Forward & GATTGCAGGTCCCAGTTCACTTC \\
\hline & Reverse & CCACTGTCCTGCTTGGACTCAC \\
\hline \multirow[t]{2}{*}{ CD68 } & Forward & GGATTCAAACAGGACCGACAT \\
\hline & Reverse & GGACACATTGTATTCCACTGCC \\
\hline \multirow[t]{2}{*}{ iNOS } & Forward & TCCTCAGGCTTGGGTCTTGTTAG \\
\hline & Reverse & TTCAGGTCACCTTGGTAGGATTTG \\
\hline \multirow[t]{2}{*}{ Arg-1 } & Forward & GCTGTGGTAGCAGAGACCCAGA \\
\hline & Reverse & CATCCACCCAAATGACGCATAG \\
\hline \multirow[t]{2}{*}{ CD163 } & Forward & CTTGGAATGGGCAAGAACAGAA \\
\hline & Reverse & TGAGTGACAGCAGAGACGCTGA \\
\hline \multirow[t]{2}{*}{ CD206 } & Forward & TGGAGTGGCAGGTGGTTAATG \\
\hline & Reverse & GGTTCAGGAGTTGTTGTGGGC \\
\hline \multirow[t]{2}{*}{$I L-1 \beta$} & Forward & AATGCCTCGTGCTGTCTGA \\
\hline & Reverse & GGATTTTGTCGTTGCTTGTCTC \\
\hline \multirow[t]{2}{*}{ IL-6 } & Forward & ATTGTATGAACAGCGATGATGCAC \\
\hline & Reverse & CCAGGTAGAAACGGAACTCCAGA \\
\hline \multirow[t]{2}{*}{ TNF-a } & Forward & TTCCAATGGGCTTTCGGAAC \\
\hline & Reverse & AGACATCTTCAGCAGCCTTGTGAG \\
\hline \multirow[t]{2}{*}{ IL-4 } & Forward & TGCACCGAGATGTTTGTACCAGA \\
\hline & Reverse & TTGCGAAGCACCCTGGAAG \\
\hline \multirow[t]{2}{*}{ IL-10 } & Forward & CAGACCCACATGCTCCGAGA \\
\hline & Reverse & CAAGGCTTGGCAACCCAAGTA \\
\hline \multirow[t]{2}{*}{ TGF- $\beta$} & Forward & CATTGCTGTCCCGTGCAGA \\
\hline & Reverse & AGGTAACGCCAGGAATTGTTGCTA \\
\hline \multirow[t]{2}{*}{$\beta$-actin } & Forward & GGAGATTACTGCCCTGGCTCCTA \\
\hline & Reverse & GACTCATCGTACTCCTGCTTGCTG \\
\hline
\end{tabular}

1:1000), mouse anti-CD163 (AbD Serotec; 1:500), rabbit anti-CD86 (ProteinTech; 1:600), rabbit anti-IL-6 (PeproTech; 1:800), rabbit anti-IL-10 (ProteinTech; $1: 600$ ), and rabbit anti- $\beta$-actin (Cell Signaling Technology; 1:1000). The blot bands were quantitated by ImageJ software (National Institutes of Health, USA). Quantitative data were expressed as the target protein $\mathrm{OD} / \beta$-actin OD ratio.

\section{Fluorescent in situ hybridization (FISH)}

Paraffin-embedded brain slices were sectioned at $4 \mu \mathrm{m}$. Fluorescence in situ hybridization was performed using custom TSG-6-specific FISH Probes (Bersinbio, Inc., Guangzhou, China). Following manufacturer instructions, brain slices were hybridized with a TSG-6 mRNA FISH Probe and labeled with Alexa Fluor Cy3 (Life Technologies, Inc., USA). Immunohistochemistry was then performed using rabbit anti-Iba-1(Abcam; 1:500), 
rabbit anti-NEUN (Abcam; 1:400), and rabbit anti-GFAP (Abcam; 1:400). Alexa Fluor 488-conjugated IgG (1:200, Invitrogen; 1:200) was applied as a secondary antibody. Photos were taken with confocal microscopes (LSM800, Carl Zeiss, Germany) following manufacturer instructions.

\section{Immunofluorescence assay}

Immunofluorescence staining was performed as previously described but with some modifications [27]. Briefly, brain sections were fixed in $4 \%$ paraformaldehyde for about $24 \mathrm{~h}$. Coronal paraffin-embedded $4 \mu \mathrm{m}$ thickness slices were conducted to Antigen retrieval and underwent blocking by $5 \%$ BSA for $1 \mathrm{~h}$. After blocking, slices were incubated overnight at $4{ }^{\circ} \mathrm{C}$ with the following primary antibodies: mouse anti-NeuN (1:100, Millipore), rabbit anti-NeuN (1:400, Abcam), mouse anti-GFAP (1:300, R\&D), rabbit anti-GFAP (1:400, Abcam), goat anti-Iba1 (1:300, Abcam), mouse polyclonal anti-TSG-6 (1:100, Santa Cruz Biotechnology), rabbit anti-CD163 (1:300, Abcam), rabbit antiCD86(1:200, R\&D), rabbit anti-pSTAT3 (1:400, Cell Signaling Technology), and rabbit anti-SOCS3 (1:500, Abcam). After washing with PBS, slices were then incubated with appropriate secondary antibodies for $1 \mathrm{~h}$ at room temperature. Following washing three times with PBS, the slices were re-stained by DAPI for $12 \mathrm{~min}$ before mounting. Then, images were obtained with a Leica DMi8 fluorescence microscope (Leica, Germany).

\section{Fluoro-Jade C (FJC) staining}

FJC staining was used to investigate neurodegeneration. Sections were subjected to FJC staining in accordance with the manufacturer instructions. Briefly, the sections were immersed in a solution of $1 \%$ sodium hydroxide in $80 \%$ alcohol for $5 \mathrm{~min}, 70 \%$ alcohol for $2 \mathrm{~min}$, distilled water for $2 \mathrm{~min}$, and followed by $0.06 \%$ potassium permanganate for $10 \mathrm{~min}$ with gently shaking. The sections were immersed in a solution of $0.0002 \%$ FJC (Millipore Corporation, USA) in $0.1 \%$ acetic acid for $30 \mathrm{~min}$. The sections were then rinsed three times in distilled water and allowed to dry at $50{ }^{\circ} \mathrm{C}$ for $15 \mathrm{~min}$ before covering with DPX medium (Sigma, USA). FJC-positive cell counting was performed as previously described but with modifications. Six sections located inside in the injured region were analyzed and FJC-positive cells were counted in each image. Data were presented by the average number of FJC-positive neurons in the fields as cells $/ \mathrm{mm}^{2}$.

\section{Microglia isolation}

Microglia of left cerebral cortex tissues with blood clots were isolated using a Percoll density gradient as described previously [32]. Briefly, brain samples obtained from each group after perfusion with $200 \mathrm{ml}$ sterile saline were dissociated with $800 \mathrm{U}$ DNase I (sigma, USA) and $7 \mathrm{ml}$ Cell Dissociation Reagent (StemPro ${ }^{\text {mx }}$ Accutase $^{\text {Tx }}$, Gibco, USA) at $37{ }^{\circ} \mathrm{C}$ for $30 \mathrm{~min}$ in an incubator. After filtration with $70-\mu \mathrm{m}$ cell strainers (BD Falcon, USA) to generate a single cell suspension, immune cells were separated by centrifugation using $40 \%$ Percoll in PBS at $1700 \mathrm{rpm}$ for $30 \mathrm{~min}$.

\section{Flow cytometry analysis}

For flow cytometry analysis of microglial polarization status in the injured brain, the isolated microglia were stained with fluorescently labeled antibodies: CD11bFITC (BD Biosciences), CD45-PE-Cy5 (BD Biosciences), CD163-APC (AbD Serotec), and CD86-PE (BD Biosciences) at $4{ }^{\circ} \mathrm{C}$ for $30 \mathrm{~min}$. Flow cytometry was performed on a FACS VERSE apparatus (BD Bioscience) and obtained data were analyzed by Flow Jo software 7.6.1(Tree Star, USA).

\section{Inflammatory cytokine measurements}

Total protein concentrations were measured using a BCA Protein Assay Kit (Genecopoeia, USA). Frozen brain samples were mechanically homogenized and centrifuged at $12,000 \mathrm{rpm}$ for $15 \mathrm{~min}$ at $4{ }^{\circ} \mathrm{C}$. The levels of interleukin-6 (IL-6), interleukin-10 (IL-10), tumor necrosis factor alpha $(\mathrm{TNF}-\alpha)$, and interleukin-1 $\beta(\mathrm{IL}-1 \beta)$ were measured using specific ELISA kits (eBIOSCIENCE, USA) according to manufacturer instructions. The concentration of the cytokines was determined by color intensity measured by spectrometry in a micro ELISA reader (Varioskan Lux, Thermo Scientific). The results are expressed as picogram per milligram for tissue samples.

\section{Statistical analysis}

All statistical analyses were performed using GraphPad Prism 6 (GraphPad software). Data are represented as a mean \pm SD. Differences between two groups were analyzed with Student's $t$ test (two-tailed), and data were analyzed by one-way analysis of variance (ANOVA) with post hoc Tukey test or Dunnett's test applied to assess multiple comparisons. Non-parametric data were analyzed using the Kruskal-Wallis $\mathrm{H}$ analysis followed by a Mann-Whitney $U$ test. Statistical significance was set at a $P$ value of $<0.05$.

\section{Results}

No significant differences on TSG- 6 mRNA expression and protein abundance were found in different timepoints in sham

We found that there was no statistical difference of TSG- 6 detected variables among sham groups at each time (Additional file 1A, B). Therefore, animals in sham group at $24 \mathrm{~h}$ after sham operation were chosen for further experiments. 
Both mRNA and protein level of TSG- 6 were upregulated after SAH injury

We first confirmed that brain TSG-6 increased in our SAH model. PCR and Western blot were performed to detect the time course of both mRNA and protein level of TSG-6 after SAH. The temporal profile of TSG-6 mRNA expression is shown in Fig. 1c. TSG-6 mRNA levels elevated immediately at $6 \mathrm{~h}$ after SAH $(p=0.0127)$ reached its peak value at $12 \mathrm{~h}$, which almost six times that of the sham group. Afterwards, mRNA levels of TSG-6 gradually declined but were still significantly increased at $24 \mathrm{~h}, 48 \mathrm{~h}$, and $72 \mathrm{~h}(P=0.0022, P=0.0001$, $P=0.0016$, respectively)

As shown in Fig. 1a, b, Western blotting revealed that the level of TSG-6 protein in the left temporal cortex increased over time. The level of TSG- 6 protein abundance was weak in the sham group, while it increased significantly at $12 \mathrm{~h}(P=0.0015)$, peaked at $24 \mathrm{~h}(P=0.0008)$, and remained ascended at $72 \mathrm{~h}$ $(P=0.0011)$ post-SAH.

\section{The endogenous TSG- 6 was mainly expressed in} microglia after SAH injury

We used FISH-ISH and double-labeling immunofluorescence to identify the cell distribution of TSG-6 at the peak activation of TSG-6 (at $12 \mathrm{~h}$ according to the PCR and $24 \mathrm{~h}$ according to the WB). FISH-ISH analysis showed that TSG-6 mRNA was expressed in microglia (Fig. 2a) at $12 \mathrm{~h}$ after SAH. Double staining confirmed that a low TSG-6 protein abundance in the microglia was observed in the sham group. Compared with the sham group, SAH augmented the relative TSG-6 protein abundance predominantly in microglia (Fig. 2c), but not neurons (Fig. 2d) or astrocytes (Fig. 2e). The co-expression of TSG-6 and Iba- 1 was obviously more after SAH (Fig. 2f).

\section{rh-TSG-6 alleviates SAH-induced brain injury (reduced brain water content and improved neurobehavioral outcomes) at $\mathbf{2 4} \mathrm{h}$ after $\mathrm{SAH}$}

At $24 \mathrm{~h}$ after $\mathrm{SAH}$, brain edema and neurobehavioral activity were examined in all groups. Two dosages of rh-TSG-6 $(1 \mu \mathrm{g} / 10 \mu \mathrm{L}$ and $5 \mu \mathrm{g} / 10 \mu \mathrm{L})$ were administrated intracerebroventricularly at $1.5 \mathrm{~h}$ after $\mathrm{SAH}$. At $24 \mathrm{~h}, \mathrm{SAH}$ insults induced poorer brain water content and neurological impairment compared to the sham group. No significant differences between $\mathrm{SAH}$ and $\mathrm{SAH}+$ vehicle groups in brain edema and neurological scores were observed. Both the two dosages of rh-TSG-6 dramatically ameliorated brain edema (Fig. 3a, b). However, only administration of high dosage rh-TSG-6 significantly improved neurobehavioral deficits (Fig. 3c) at $24 \mathrm{~h}$ post-ictus $(P=0.0022)$ vs. $\mathrm{SAH}+$ vehicle, $n=6$. These results indicated that a high dosage is effective for
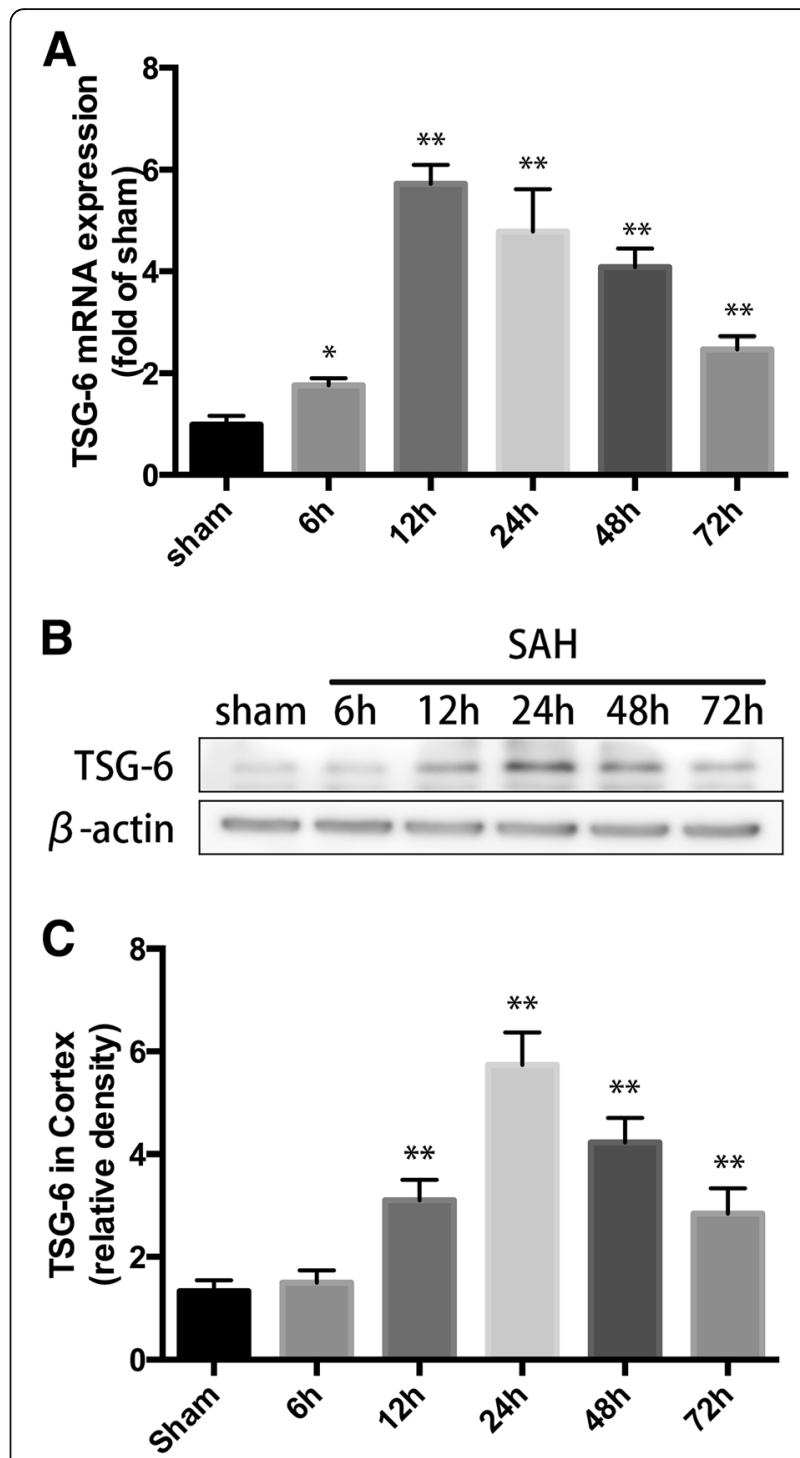

Fig. 1 Endogenous expression of TSG-6 in brain tissue after subarachnoid hemorrhage (SAH). a Quantification of TSG-6 mRNA level in rat temporal cortex. $\mathbf{b}$ Western blot analysis showed the level of TSG-6 protein abundance at 6, 12, 24, 48, and $72 \mathrm{~h}$ after SAH. c Quantification of the TSG-6 protein level as shown in $\mathbf{b}$. All values are presented as means \pm SD, $n=6$ in each time point per group. ${ }^{*} p<0.05,{ }^{* *} p<0.01$ versus sham group

reducing EBI; thus, a high dosage was selected for further studies.

rh-TSG-6 treatment attenuated SAH-induced brain cell injury

Neural loss is a key event in EBI after SAH. As shown in Fig. $4 \mathrm{a}$, the procedure of $\mathrm{SAH}$ induced a large amount of neurodegeneration as revealed by FJC staining. Furthermore, there was no significant difference between the $\mathrm{SAH}$ and $\mathrm{SAH}+$ vehicle groups. When compared with the vehicle group at $24 \mathrm{~h}$ after $\mathrm{SAH}$, the number of $\mathrm{FJC}^{+}$ 


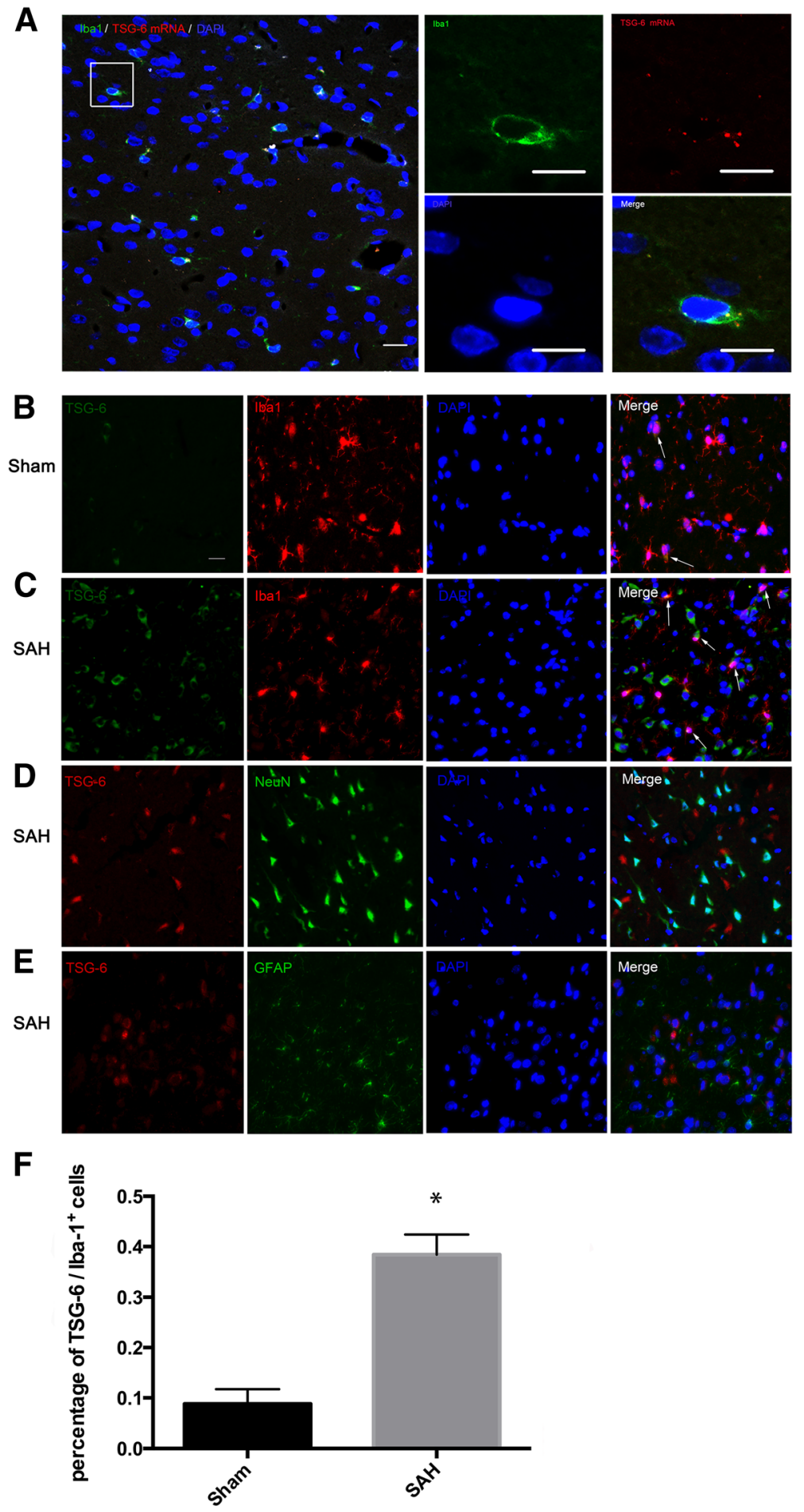

Fig. 2 Spatial distributions of endogenous TSG-6 after SAH. a After injury, TSG-6 mRNA expression was clearly seen in microglia (Iba1 ${ }^{+}$cells). $\mathbf{b}$ Representative immunofluorescence staining slices of TSG-6 with calcium-binding adaptor molecule 1 (Iba1) in sham animals. c More Iba1positive cells were observed after SAH when compared with sham group. Negative colocalization of TSG-6 with neurons (NeuN) (d) and astrocytes(GFAP) (e) at $24 \mathrm{~h}$ following SAH. Arrows point to TSG-6-positive microglia. $n=6$ in each group. ${ }^{*} p<0.05$ versus sham group. Scale bars $=20 \mu \mathrm{m}$. Bars in higher magnification panels are $10 \mu \mathrm{m}$ 

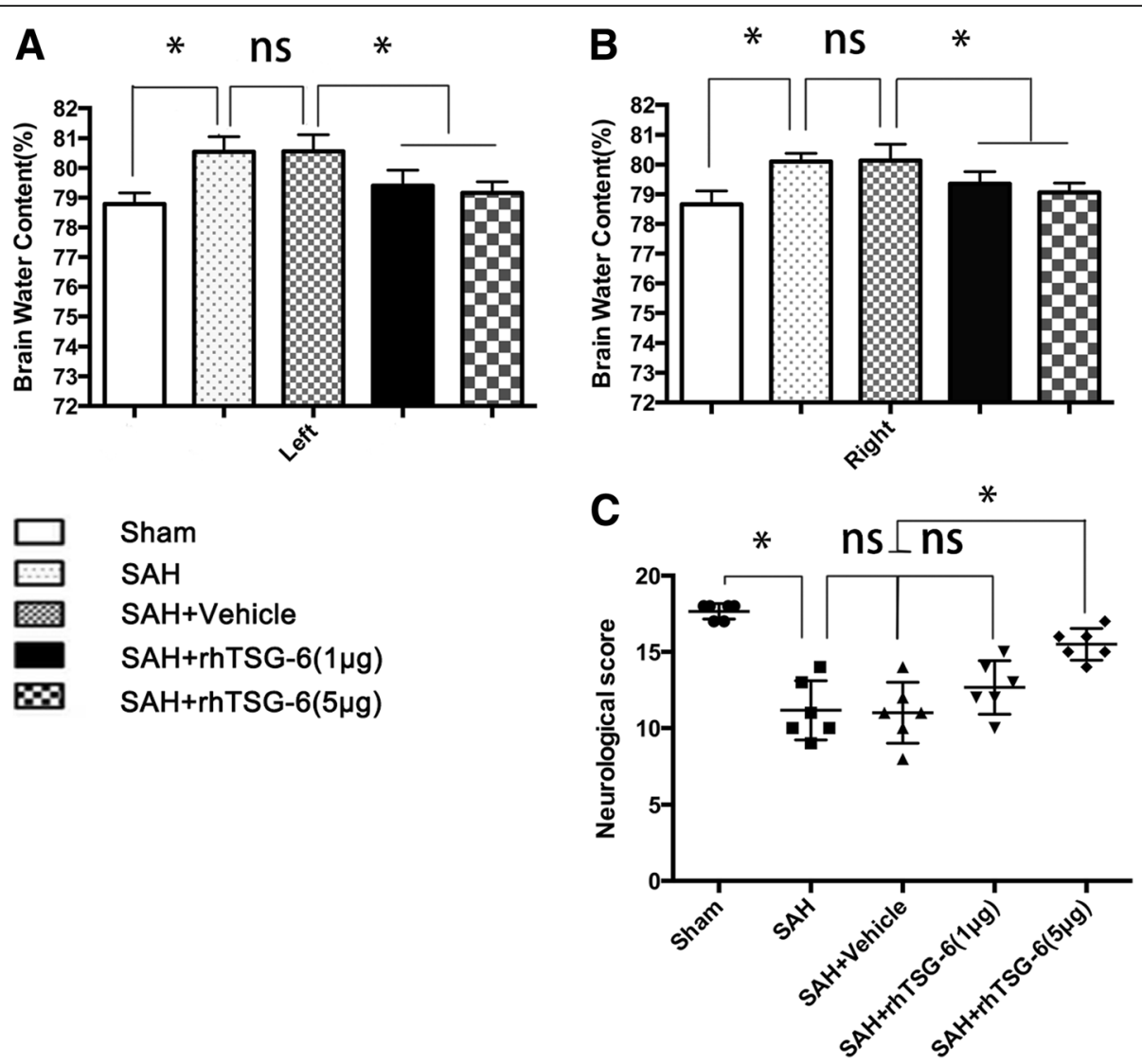

Fig. 3 Effects of rh-TSG-6 treatment on brain edema $(\mathbf{a}, \mathbf{b})$ and neurobehavioral deficits $(\mathbf{c})$ at $24 \mathrm{~h}$ after SAH. Brain water content using a wet/dry weight method was measured at $24 \mathrm{~h}$ after SAH. Neurological scores were recorded at $24 \mathrm{~h}$ after SAH. High dosage of rh-TSG-6 effectively reduced brain edema $(\mathbf{a}, \mathbf{b})$ and improved neurological functions $(\mathbf{c})$ at $24 \mathrm{~h}$ following SAH. Values are expressed as the mean $\pm S D, n=6$ in each group. ${ }^{*} P<0.05$

cells was significantly decreased in the injured cortical region in rh-TSG-6-treated SAH rats $(P=0.0022)$ (Fig. 4a, b). Furthermore, the rh-TSG-6 group had a lower number of Fluoro-Jade $\mathrm{C}$-stained neurons than the SAH-vehicle group at $72 \mathrm{~h}$ after SAH $(P=0.0002$, Additional file 2A).

\section{Knockdown of endogenous TSG- 6 aggravated neural death and neurologic deficits after SAH}

To further explore the role of TSG-6 in the pathogenesis of EBI, knockdown of endogenous TSG-6 was performed. TSG-6 siRNA was injected into the cerebroventricle at $48 \mathrm{~h}$ before $\mathrm{SAH}$ induction. Interfering efficiency of TSG-6 siRNA was verified using qPCR and WB experiments. Neurologic score evaluation was also performed $24 \mathrm{~h}$ after SAH. As shown in Fig. 5a-c, scramble siRNA had no effect on endogenous TSG-6 mRNA and protein expression compared to the SAH group, but knockdown of endogenous TSG-6 with siRNA decreased the mRNA and protein level of TSG-6 in the brain $(p=0.0001$ and $p=0.0004$, respectively; $n=6 ; n=6$ ). Furthermore, knockdown of endogenous
TSG-6 significantly aggravated neurologic deficits $24 \mathrm{~h}$ after SAH (Fig. 5d).

\section{SAH-induced activation of microglia and microglial M1 phenotype polarization was dominated during the SAH early phase}

Activation of microglia is a hallmark of neuroinflammation. In this study, we investigated the characteristics of microglial activation in the early stages following SAH induction. We first detect whether microglia were markedly activated after SAH. Microscopic examination of whole brain sections stained for Ibal confirmed that SAH-induced microglia were dramatically activated for whole brain sections compared to the sham group (Additional file 3). Next, we detected mRNA levels of markers of M1/M2 microglial cells and M1/M2-associated cytokines at corresponding times post-SAH. The data showed M1-associated markers CD68, CD86, and iNOS increased immediately and peaked at $24 \mathrm{~h}$ (iNOS) and $48 \mathrm{~h}(\mathrm{CD} 68, \mathrm{CD} 86)$ and remained at high levels for 72 h. M2-associated markers increased slowly and peaked at $48 \mathrm{~h}$ (CD206) and $72 \mathrm{~h}$ (Arg-1, CD206) and 


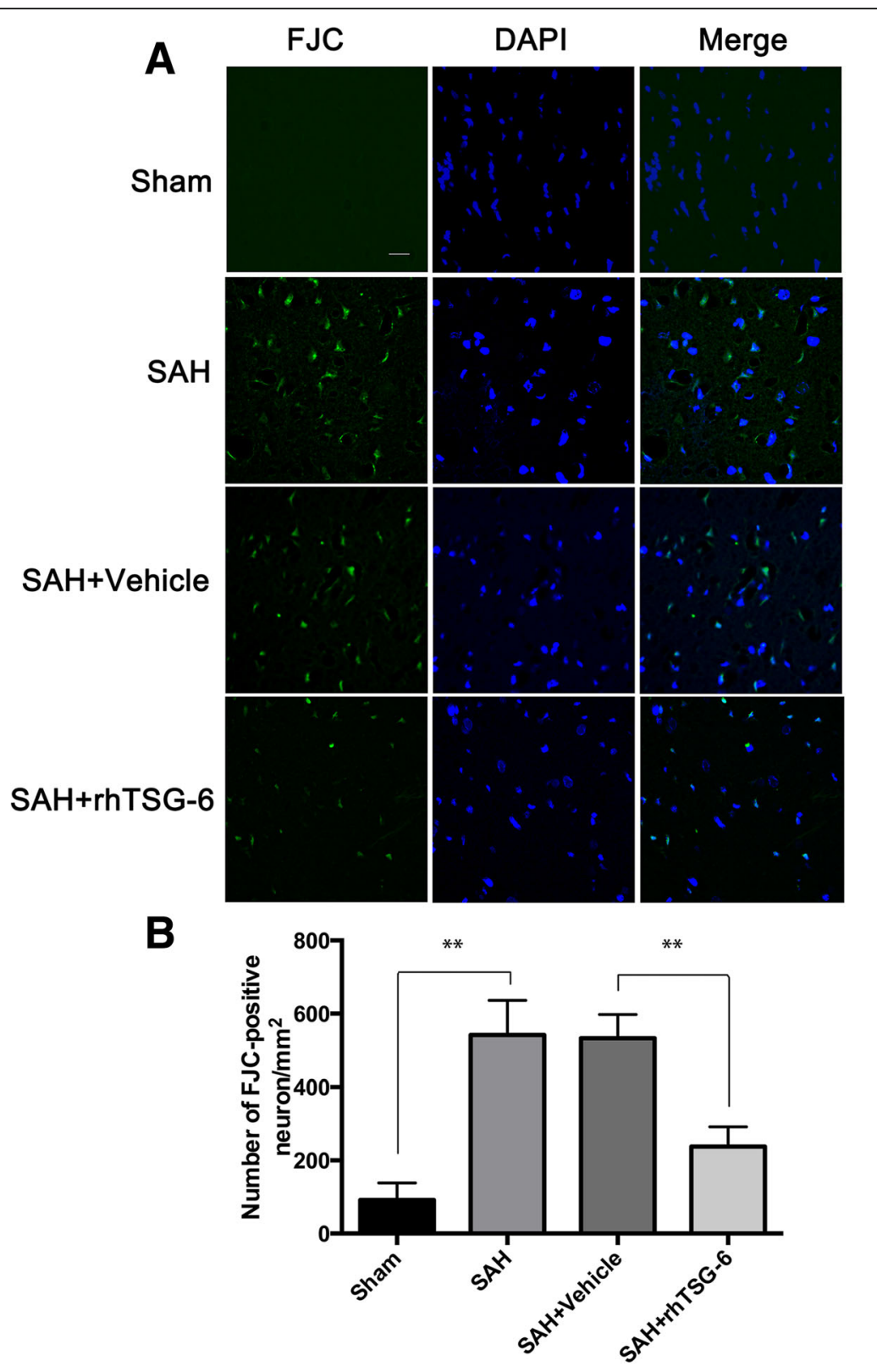

Fig. 4 Effects of rh-TSG-6 on neuronal degenerating at $24 \mathrm{~h}$ after subarachnoid hemorrhage (SAH). a Representative microphotographs of FluoroJade C staining (FJC)-positive neurons in the Sham, SAH, Vehicle and rh-TSG-6 groups at $24 \mathrm{~h}$ following operation. b Quantitative analysis of FJCpositive cells was performed at the ipsilateral cortex. $n=6$ in each group. Data are expressed as mean \pm SD. ${ }^{*} P<0.01$. Scale bar $=20 \mu \mathrm{m}$

maintained a slight growth within $72 \mathrm{~h}$ after SAH (Fig. 6a).

Consistent with the M1/M2 marker qPCR results, mRNA expression levels of both pro-inflammatory and anti-inflammatory cytokines showed similar trends. Generally, the pro-inflammatory cytokine expression levels had a striking burst and peaked at $6 \mathrm{~h}$ (IL-1 $\beta$ ) or $24 \mathrm{~h}$ (TNF- $\alpha$, IL-6) post-SAH. In contrast, expression of anti-inflammatory cytokines was not augmented significantly until 6 h (TGF- $\beta$ ) or 24 h (IL-4, IL-10) and maintained a relatively gentle increase (Fig. 6b).
These results demonstrated that SAH induced a remarkable increase in both M1- and M2-type mRNA expression in the injured brains of rats compared with the sham groups. Moreover, SAH-induced activated microglia were mainly polarized to the M1 phenotype.

TSG-6 treatment provides neuroprotection in the brain under SAH conditions by modulating microglial phenotype and decreasing its pro-inflammation activity Polarization of microglia was involved in the pathological progression of neurotoxic insults and strokes. To 


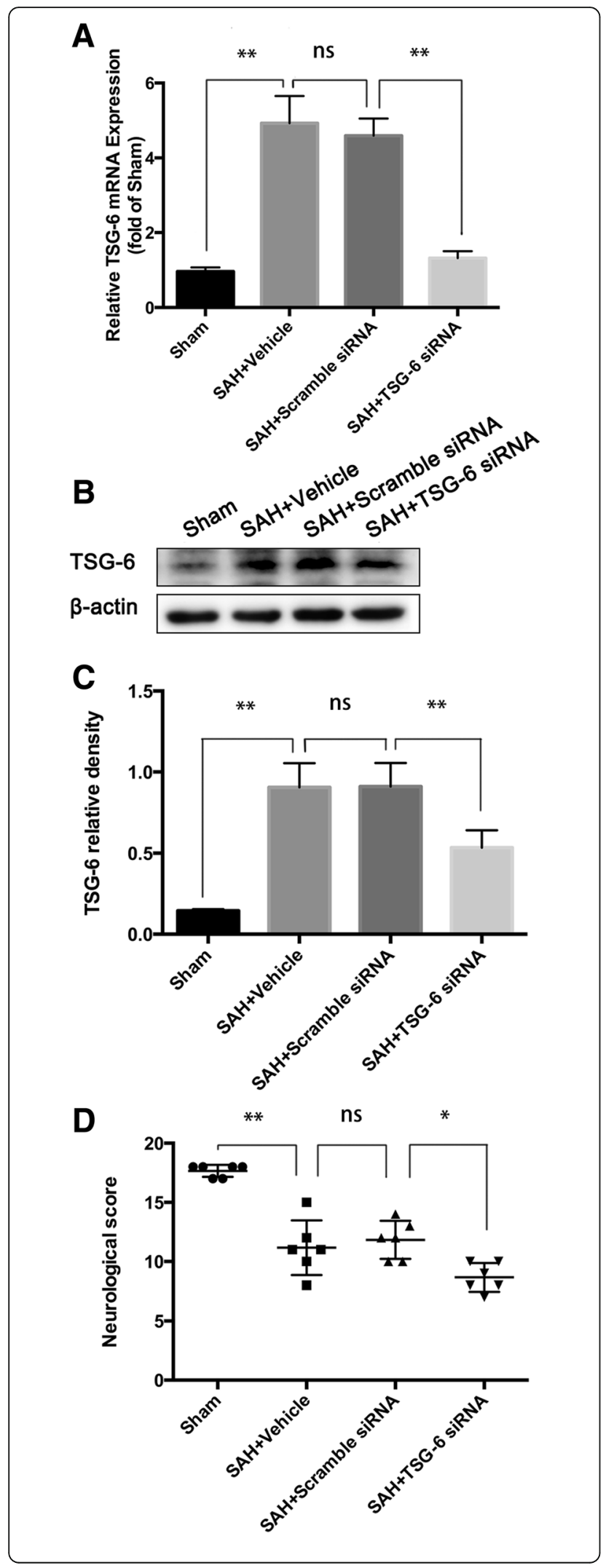

Fig. 5 Effects of TSG-6 siRNA pre-treatment in SAH rats. The expression of TSG-6 at mRNA (a) and protein (b) level at $24 \mathrm{~h}$ after intracerebroventricular injection of TSG-6 siRNA. c Neurologic score evaluation was also performed at $24 \mathrm{~h}$ after SAH. Knockdown of endogenous TSG-6 significantly worsened neurologic deficits at $24 \mathrm{~h}$ after SAH. ( $n=6$ rats/group). Values are reported as means \pm SD. ${ }^{*} p<0.05 ;{ }^{* *} p<0.01$

assess whether TSG-6 has a biological role in microglial polarization, the ipsilateral temporal lobe cortex was subjected to flow cytometry for the ratio of M1- and M2-type microglia measurement. Using flow cytometric analysis [33], we distinguished between activated microglia $\left(\mathrm{CD} 11 \mathrm{~b}^{+} \mathrm{CD} 45^{\mathrm{low}}\right)$ and infiltrating macrophages $\left(\mathrm{CD} 11 \mathrm{~b}^{+} \mathrm{CD} 45^{\text {high }}\right)$ and detected changes in classical activation marker CD86 and alternative activation marker CD163 expression in specific cell populations. The gating strategy is shown in Fig. 7a. As shown in Fig. $7 \mathrm{~b}$, compared with the sham groups, the SAH and vehicle-treated group showed a higher percentage of $\mathrm{CD}_{11} \mathrm{~b}^{+} \mathrm{CD} 45^{\text {low }}$ microglia labeled with markers for M1 (CD86) and M2 (CD163) phenotypes. In rats treated with rh-TSG- 6 after SAH induction, the expression of CD163 was increased on $\mathrm{CD}_{11} \mathrm{~b}^{+} \mathrm{CD} 45^{\text {low }}$ microglia than those in the vehicle treated group, while the expression of M1 marker (CD86) exhibited a remarkable attenuation. In accordance with declined M1 polarization elevating M2 phenotype after rh-TSG-6 treatment, there was a remarkable attenuation of TNF- $\alpha$ expression level and upregulated IL-10 expression level measured by ELISA in the rh-TSG- 6 treatment group. As expected, no significant changes of either M1 or M2 ratio were noticed between the SAH and vehicle-treated groups.

Overall, these results demonstrated that rh-TSG-6 treatment partially retained SAH-driven M1 polarization and skewed the balance of M1/M2 ratio to a beneficial phenotype, thereby conferring its neuroprotective properties.

\section{Deficiency of endogenous TSG-6 leads to an exaggerated pro-inflammatory microglial phenotype}

To further determine the role of TSG- 6 in modulatory effects of triggering phenotypic conversion of microglia, mice were subjected to TSG- 6 siRNA treatment $48 \mathrm{~h}$ before SAH induction. The results of double immunofluorescent staining and western blot showed that the M1-associated marker CD86 (Fig. 8a, b, e) and M2-associated marker CD163 (Fig. 8c, d, f) were both significantly upregulated $24 \mathrm{~h}$ after $\mathrm{SAH}$ compared to the sham group. When TSG- 6 was suppressed, the induction of SAH potentiated the protein expression of CD86, while the expression of M2 marker CD163 in Iba1 was lower than the scramble siRNA-treated group. 


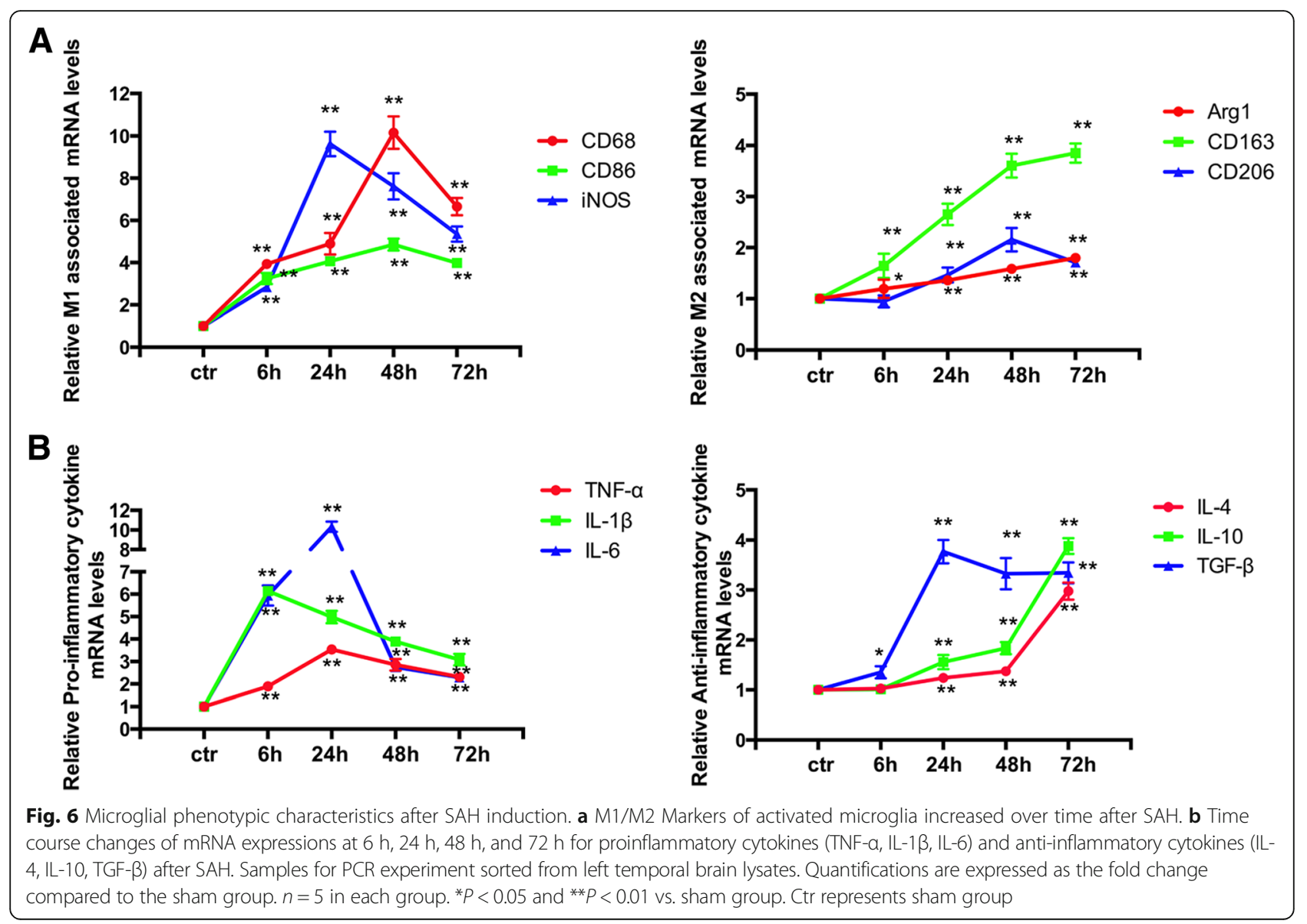

Considering the above results, we concluded that a loss of TSG-6 triggers microglial phenotypic changes with a bias towards pro-inflammatory activity, namely amplifying M1 polarization of activated microglia.

Effects of TSG- 6 were involved in impeding the procedure of phosphorylation and the cellular location of Stat3

STAT3 is an important transcription factor associated with microglial polarization status. Overactivation of p-STAT3 can contribute to the upregulation of proinflammatory cytokines under some kinds of stroke. Since the activity of pSTAT3 was observed mainly in microglia/macrophages, but not in either neurons or astrocytes, therefore, immunofluorescence determined the expression of p-STAT3 in microglia in the rat cortex following SAH at $24 \mathrm{~h}$. As shown in Fig. 9, fluorescence intensity of p-STAT3 was barely detected in the sham group. Obviously, rats which underwent SAH exhibited significantly increased p-STAT3 levels compared with sham-operated rats. Next, intervention with rh-TSG-6 diminished the level of phosphorylation of STAT3 in the SAH group. Correspondently, phosphorylation level of p-STAT3 was more enhanced in TSG-6 siRNA-pretreated rats. In addition, a reduction in nuclear translocation of STAT3 following rh-TSG-6 treatment and p-STAT3 immunoreactivity were more elevated following silencing treatment. Therefore, these results suggested that TSG- 6 may be associated with the phosphorylation of STAT3.

The effects of TSG- 6 on modulating microglial phenotype was involved in SOCS3/STAT3 pathway

Among the candidate signal pathways, the STAT3 signaling pathway can play a pivotal role in neuroinflammation and the activation of microglia within CNS. To further explore the underlying mechanism of TSG- 6 in regulating microglia activation, factors in the STAT3 pathway pivotal to microglia polarization were tested. Results of Western blot (Fig. 10a) showed that after $\mathrm{SAH}$, inflammatory responses occurred. Significant activation of STAT3 was observed accompanied with elevated expression of IL- 6 rather than IL-10 and a raised SOCS3 levels in comparison with the sham group. However, the induction of SAH or TSG-6 was not observed to change the protein level of STAT3. Additionally, post-SAH TSG-6 upregulation was associated with an increase in IL-10 and SOCS3 levels and a decrease in 

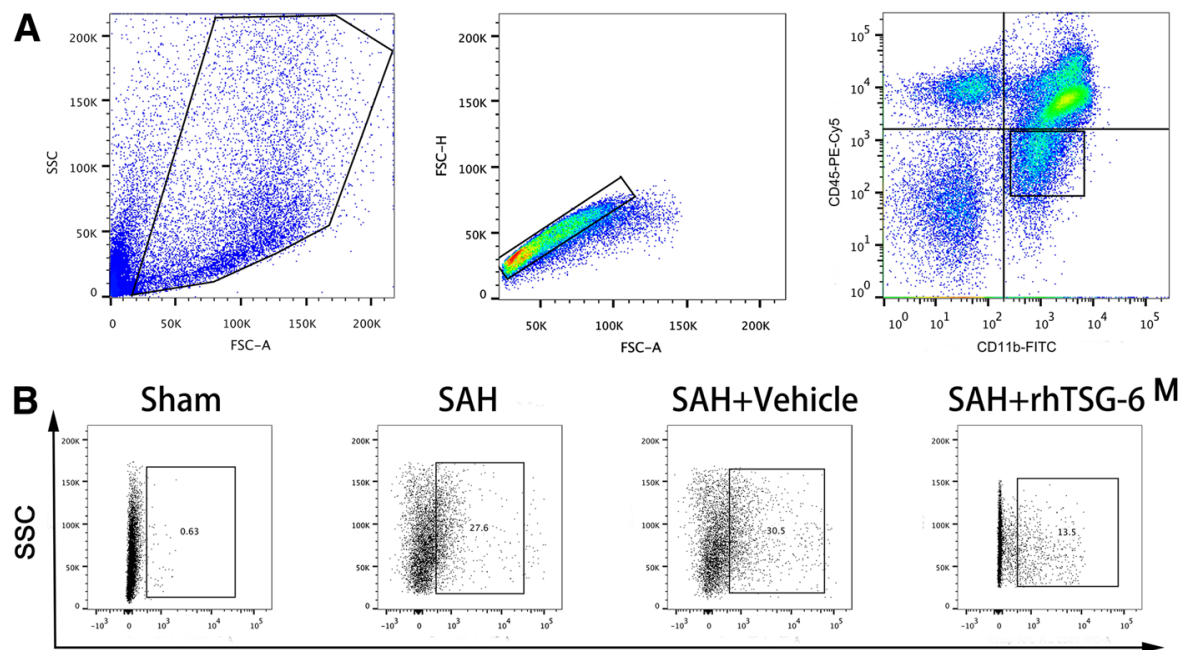

$\mathrm{SAH}+$ Vehicle

SAH+rhTSG-6 M1
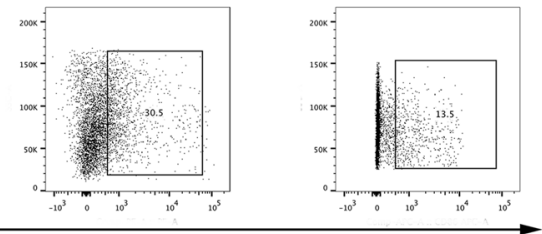

CD86-PE

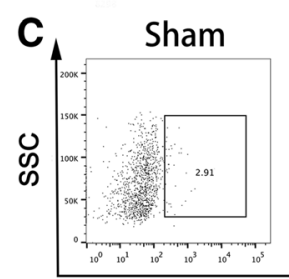

$\mathrm{SAH}$

SAH+Vehicle

SAH+rhTSG- $6^{\mathrm{M} 2}$

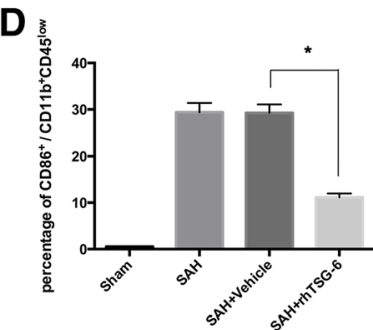

$E_{\frac{z}{n}}$

CD163-APC
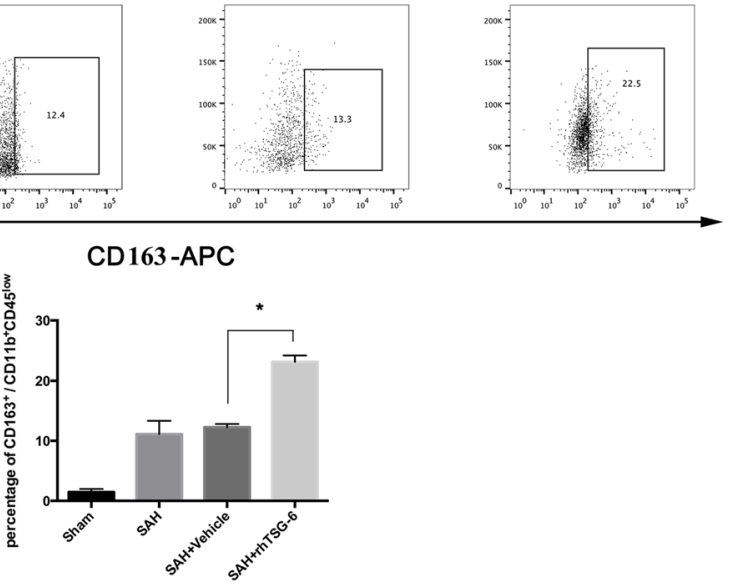

F
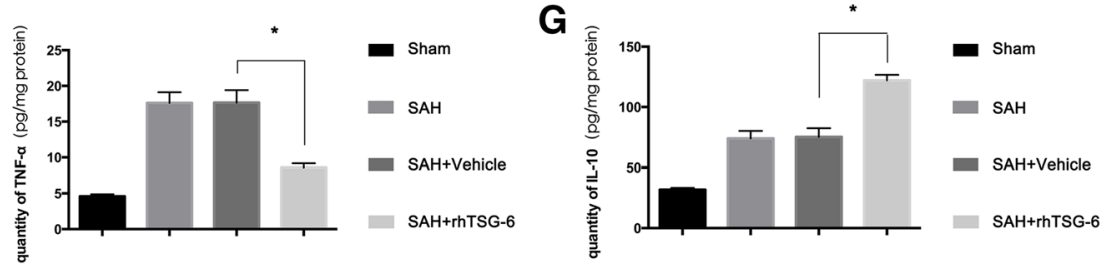

Fig. 7 Effects of rh-TSG-6 on SAH-induced microglia polarization. a Representative FACS plots showing gating strategy we use in flow cytometry analysis. Cells populations in the right dot plots defined as CD11 $\mathrm{b}^{+} \mathrm{CD} 45^{\text {low }}$ (microglia) were gated for further analysis. Representative dot plots

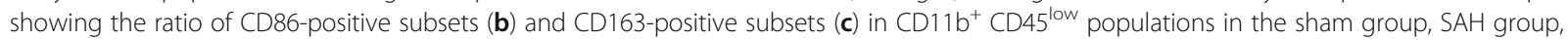

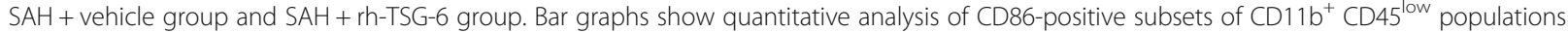
(M1 microglia) (d) and CD163-positive subsets of CD11 b+ CD45 low populations (M2 microglia) (e) in different groups. Inflammatory mediators expressed in the brain cortex after SAH were detected with ELISA. Tissue TNF- $a$ and IL-10 concentrations were analyzed from samples and standards in duplicates and expressed as pictograms per milliliter. rh-TSG-6 administration obviously reduced the level of TNF-a (f) and increased the level of IL-10 (g) in the brain tissue. Samples for FACS and ELISA experiments sorted from tissue lysates of left temporal lobe of the brain. The values (means \pm SD) are representative of three independent experiments. ${ }^{*} p<0.05 . n=6 /$ group. SSC $=$ side scatter channel, FSC-A = forward scatter channel area, FITC = fluorescein isothiocyanate, PE = phycoerythrin, and APC = allophycocyanin

IL-6 and phospho-STAT3 levels in brain tissues $24 \mathrm{~h}$ after SAH. IL-6-mediated activation of STAT3 was inhibited in injured brain tissues of SAH rats treated with rh-TSG-6.
Finally, we knocked down endogenous TSG-6 to test the association between TSG- 6 and the SOCS3/STAT3 axis (Fig. 10b). Conversely, deficiency of TSG-6 in SAH rats showed more increased pSTAT3 compared to 


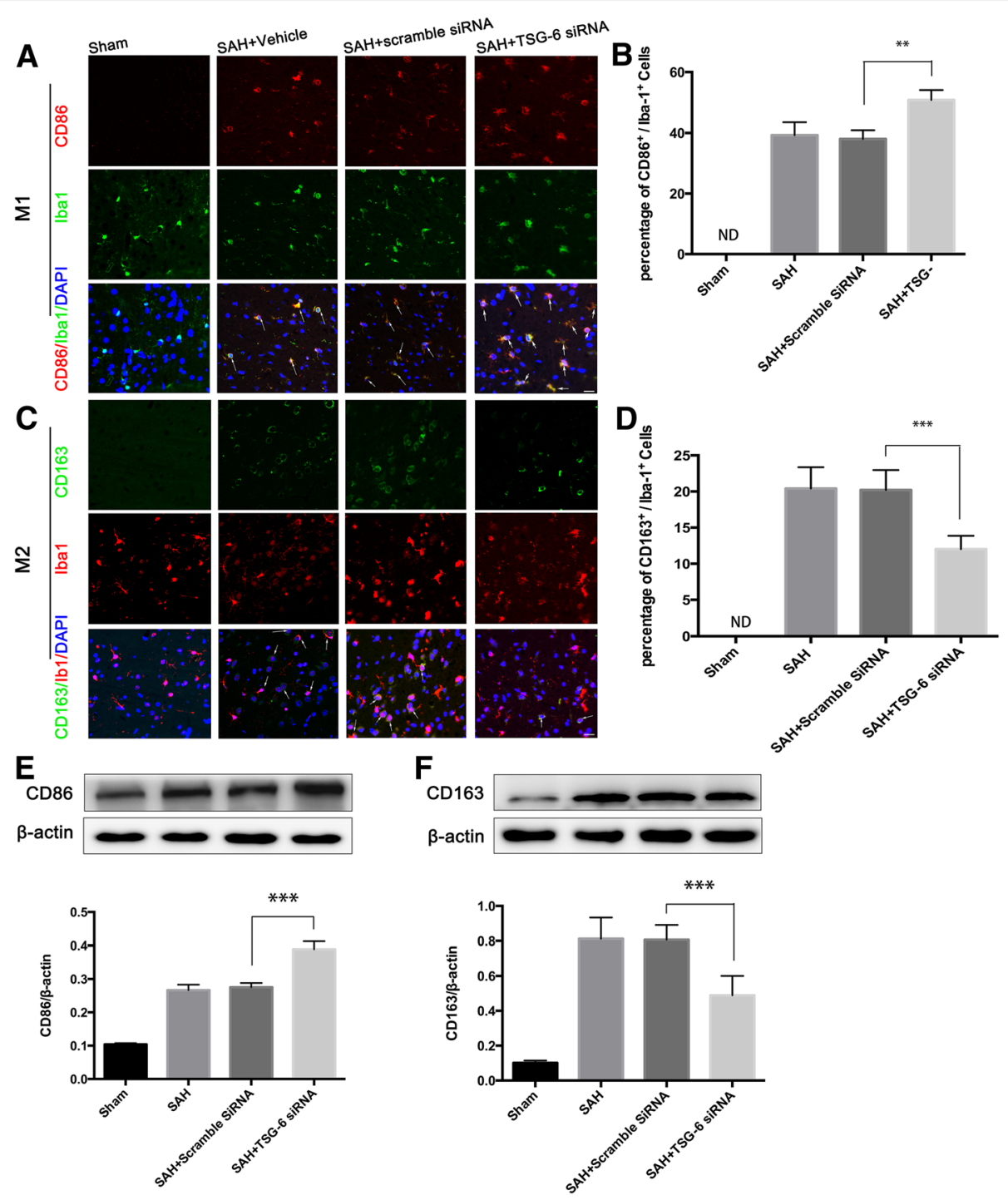

Fig. 8 Deficiency of endogenous TSG-6 leads to an exaggerated proinflammatory microglial phenotype. Immunofluorescence labeling and quantification analysis (a-d) and western blot $(\mathbf{e}, \mathbf{f})$ and showing that inhibition of TSG-6 further enhanced the increased levels of CD86 and the enhanced CD163 levels was decreased $24 \mathrm{~h}$ after SAH compared with the scramble siRNA group. The samples for western blot are tissue lysates obtained from the left temporal lobe of the brain. White arrows indicate typical cells. Values of the relative densitometric analysis are expressed as mean $\pm \mathrm{SD}$, Scale bar $=20 \mu \mathrm{m}, n=6$ in each group. ${ }^{* *} P<0.01 ;{ }^{* *} P<0.001$

scramble siRNA treated rats. As expected, the two inflammatory cytokines indicated opposite trends (amplifying IL-6 expression and declining IL-10 expression) contributed by TSG-6 silencing treatment. Expression of SOCS3 showed a weaker response to SAH insult. Therefore, these results verified TSG-6 functions in altering microglial phenotypes by modulating SOCS3/STAT3 axis expression in inducible SAH rat model.

\section{Discussion}

In this study, we revealed a neuroprotective effect with emphasis on targeting the immunomodulatory functions afforded by TSG-6 in a rat model of SAH. Our data demonstrated that TSG-6 was upregulated in the brain cortex in a time-dependent manner after SAH. To further determine the role of TSG-6 following SAH, we continued our research in experimental rats. Exogenous TSG-6 greatly attenuated neurological deficits and brain water under inducible subarachnoid hemorrhage in vivo. After silencing endogenous TSG-6, early brain injury was further exacerbated. These results indicated that the neuroprotective effect of TSG-6 is associated with phenotypic modulation of the microglia shift. Signaling protein expression-associated STAT3 in brain tissue after SAH were investigated. The finding showed that levels of TSG-6 were involved in STAT3 


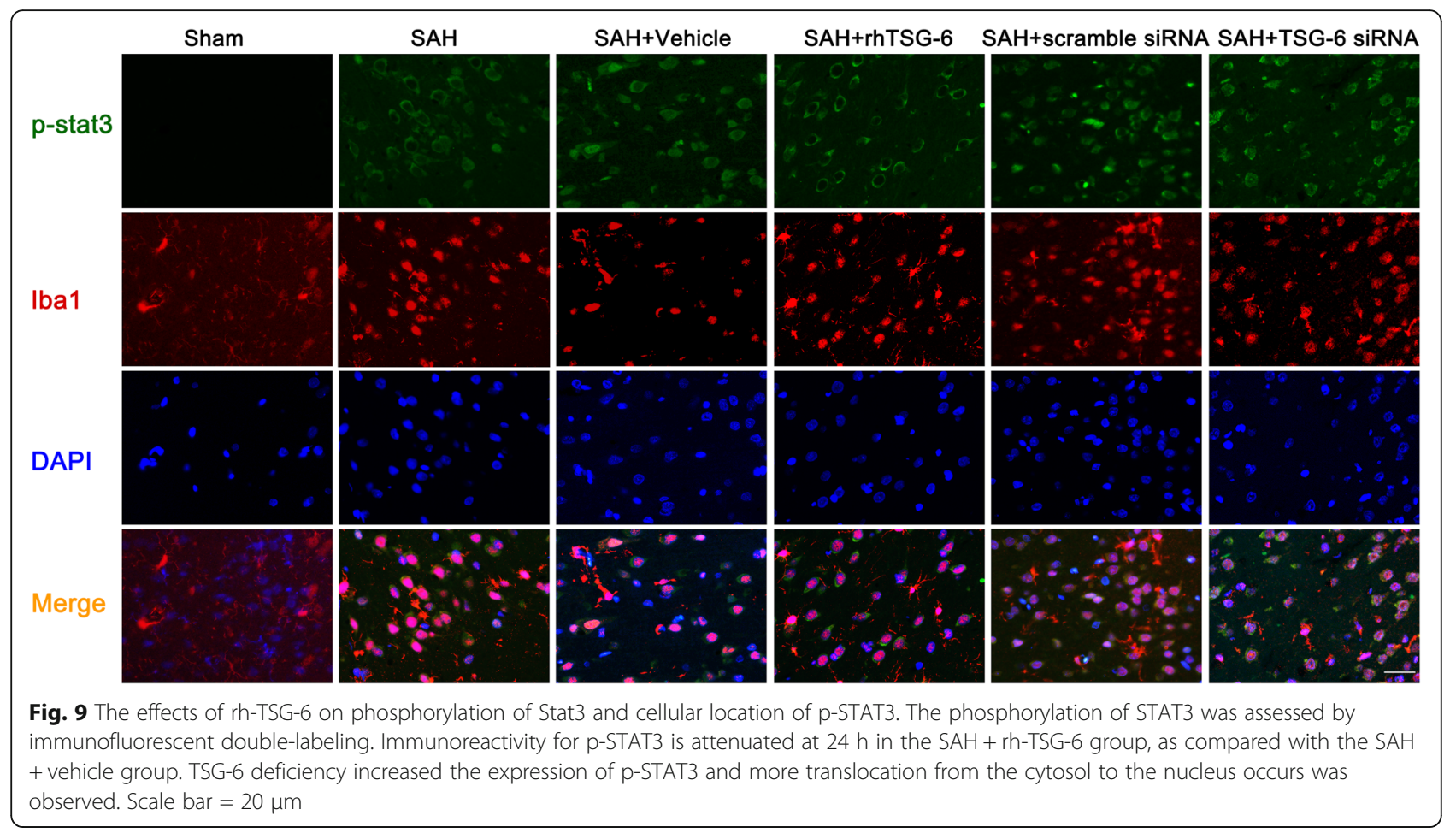

phosphorylation, which was an important factor for controlling microglial inflammatory subtypes. Accordingly, these results suggested that TSG-6 may play an endogenous brain protection role after SAH and an enhanced expression or supplementation of TSG-6 may induce a selective modulation of microglia polarization to anti-inflammatory phenotypes via the SOCS3/STAT3 pathways (Fig. 10).

TNF-stimulated gene-6 (TSG6) is a pleiotropic immunomodulatory protein activated rapidly in response to stimuli and functions at an early phase of inflammatory procedure. The protective effects of TSG- 6 have been well studied in the brain, most of which focused on the anti-inflammatory aspect, especially its role in inhibiting activation of microglia/macrophages [27, 28, 34]. Given that the suppression of microglia/macrophages may also compromise the normal physiological defense mechanism of CNS and induce inevitable side effects $[35,36]$, the mechanism by which TSG- 6 confers neuroprotection requires further investigation. In this study, the protein of TSG-6 was almost located in $\mathrm{Iba1}^{+}$microglia. However, this result was different from Coulson-Thomas et al.'s work, in which they found TSG-6 was only located in astrocytes in a SCI rat model [37]. As indicated in Fig. 2c, TSG-6 staining is positively co-expressed in not only microglia but also other cell types as well. According to the literature, blood-derived immunocytes, such as neutrophils and DC cells, can express TSG-6 under inflammation stimulation [26]. Therefore, under the circumstance that BBB is no longer intact after SAH insult,
TSG-6 could originate from infiltrating peripheral immunocyte. Indeed, we cannot exclude the possibility that TSG-6 expressed by other cells also plays a role, but the proportion of infiltrating peripheral immune cells is relatively small compared to resident microglia, especially in the early stages $[38,39]$. Thus, we assumed that data detected in the current study mainly reflects the biological function of microglial TSG-6. Correspondingly, by intervening in the concentration of TSG-6 (exogenous supplementary or knockdown), our results indicated that the level of microglial TSG-6 was closely associated with the severity of brain injury after $\mathrm{SAH}$, which was in lines with several other studies that the level of TSG-6 can reflect the disease progression and act as a novel prognostic factor [40].

Some may argue that simply dividing microglia into the supposed dichotomy between M1 and M2 phenotypes is oversimplified [41]; however, this classification may provide a bridge for understanding the function of microglia in several brain diseases [24]. When irritated proinflammatory-status microglia dominates the inflammatory microenvironment, inflammation-induced developmental brain injury can be observed in some CNS diseases $[42,43]$. Consistent with the findings of previous study [44], our study showed that, after induction of $\mathrm{SAH}$, augmented microglia were identified by the panorama of brain slices and more strikingly elevated proinflammatory genes compared to anti-inflammatory genes within $72 \mathrm{~h}$ after SAH was revealed by PCR. rh-TSG-6 treatment significantly suppressed the prevailing M1 


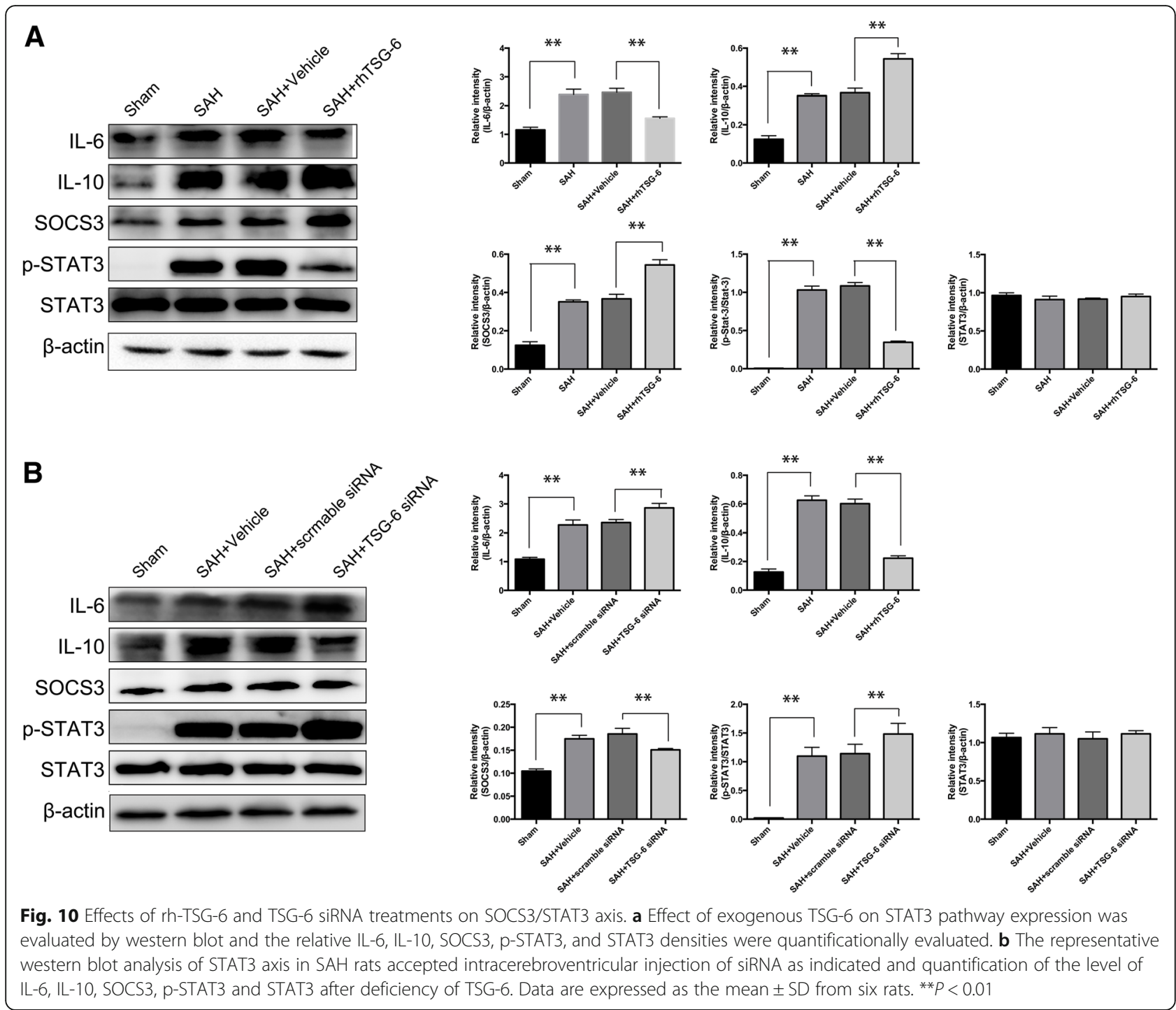

phenotypes and observed a trend towards M2 phenotypes (Fig. 4). Resident microglia are major cells involved in inflammatory processes in the brain and are first to respond to the disturbed brain environment $[38,45]$. Yet the time for microglia-mediated inflammation response to manifest occurred over hours to days, thereby creating an opportunity for clinical therapeutic intervention [46]. Accordingly, early limitation of microglia skewing towards proinflammatory phenotypes can help to preserve brain tissue. As expected, a lower abundance of endogenous TSG- 6 protein led to the provocation of M1 phenotypic microglia after SAH and to be concomitant with the marked proinflammatory environment are degenerated neurons and upcoming unfavorable outcomes, while after administration of rh-TSG-6, the modified inflammatory milieu at the early stage of EBI through M1/M2 switching was accompanied by an improved outcome. A timely shift to beneficial M2 microglia can resolve neuroinflammation and create a microenvironment friendly to CNS repair [21]. However, long-term blockage of inflammation via suppressing M1 activation may not induce overall beneficial effects because deficiency of M1 phenotypic microglia will hinder clearing cell debris so as to prolong the inflammatory process. It is clear that cessation of inflammation progression before mounting to its peak is effective in alleviating brain injury. Accordingly, in the present study, we observed the effect of acute rh-TSG- 6 treatment on early outcomes $(24$ h) in SAH rats. Modulation of the M1/M2 balance by TSG-6 is protective against neuronal injury $24 \mathrm{~h}$ after SAH. This effect also has a beneficial impact on neuronal survival at $72 \mathrm{~h}$ after $\mathrm{SAH}$ as indicated by our FJC data. Collectively, these results indicated a causal relationship between TSG- 6 and the M1/M2 microglia switch. 
Controlled neuroinflammation and immune cell infiltration within injured CNS were supposed to serve protective and beneficial functions [47]. However, to what extent and to what time inflammatory processes are deleterious and/or beneficial to brain recovery remain controversial. Therefore, a more precision modulation of microglial inflammation response remains to be explored. To date, there exists much uncertainty over which intracellular signaling pathways are involved in the mediation of the microglial shift in SAH. It is reported that STAT family members play various roles in microglial and macrophage polarization [48]. Our study discovered that microglial STAT3 was highly activated in the hemorrhagic hemisphere after experimental SAH. However, disparate functional outcomes from activated STAT3 were observed in different cerebral pathological conditions. Furthermore, whether microglial STAT3 activation during a pathologic condition results in inflammation or neuroprotection remains relatively controversial $[49,50]$. Recent studies have shown that upstream kinase can regulate STAT3 and the subsequent downstream genes regulated by STAT3 [51]. Specifically, it has been demonstrated that both the proinflammatory mediator IL- 6 and the anti- inflammatory cytokine IL-10 share the same STAT3 pathway [52]. Depending on different stimuli, IL-10-mediated STAT3 responses can lead to an anti-inflammatory phenotype associated with a higher expression of M2 neuroprotective markers [53]. However, under some sublethal strikes, a loss of normal feedback modulation on STAT3 from SOCS3 can result in overactivation of STAT3, thereby favoring IL-6 driven STAT3 $[54,55]$. In good agreement with the previous studies [56], our results demonstrated that overactivation of STAT3 in microglia is detrimental, and a reduced phosphorylation of STAT3 may provide anti-inflammatory effects.

Regarding the possible mechanisms underlined in TSG-6-conferred neuroprotection, we discovered that treatment with rh-TSG-6 following SAH effectively decreased the expression of p-STAT3 accompanied by the increased of expression of SOCS3 as well as IL-10 and a decreased IL- 6 expression related to the vehicle-treatment group, both of which correlated with a dominated anti-inflammatory microenvironment. In vivo TSG-6 knockdown further aggravated STAT3 activation and reversed the effects of IL-6, IL-10, and SOCS3 expression. As a result, a pronounced polarization to M1 phenotype microglia was linked with worsened brain injury, which is consistent with several previous works $[23,57]$. Taken together, we speculated that TSG-6 appeared to play a unique role in M1/M2 polarization by modulating IL-6/STAT3 or/and IL-10/ STAT3 activity through SOCS3 activation.

Recently, a study from Mittal et al. supported our finding whereby TSG-6 inhibited aberrant activation of STAT3 and primed the innate immunity response from a state of generating proinflammatory cytokines to a distinct anti-inflammatory status [58]. Although we have demonstrated that TSG-6 confers its neuroprotection partly depending on the SOCS3/STAT3 axis, the detailed mechanisms underlined require further investigation. Additionally, this study mainly focused on the role of TSG-6 in the early stage of SAH injury. The potential long-term roles of TSG-6 on M1/M2 phenotypic balance and responses to brain injury are not addressed herein and should be investigated in the future studies. Admittedly, injury-recovery is a continuously variable process. All immunotherapies may not achieve optimal clinical transformation if they do not compliment changes during the overall process. Likewise, whether the effect of TSG- 6 beyond the EBI at a later time satisfies the demands of the CNS microenvironment will impact the recovery processes and the final neurological state. However, due to limitations inherit in the puncture SAH model, it is difficult to explore this research topic. A more perfect SAH model which is suitable for long-term research on SAH pathophysiological conditions is required.

\section{Conclusions}

In summary, we identified the protective effects of TSG-6, a novel endogenous anti-inflammation protein, after $\mathrm{SAH}$ insults by inhibiting inflammation through the SOCS3/STAT3 pathway. It was discovered that exogenous administration of rh-TSG-6 can provide neuroprotective effects related to modulating microglial polarization phenotypes. Our finding suggested that TSG-6 is a promising candidate for alleviating the degree of EBI following SAH, which may expand application in targeting microglial overactivation-mediated inflammation in cerebrovascular diseases.

\section{Additional files}

\begin{abstract}
Additional file 1: Analysis of levels of TSG-6 gene and protein in different time-points in the sham ( 6 h, 12 h, 24 h, 48 h, 72 h). No significant differences of TSG-6 gene (A) and protein (B) were found among different groups. All values are presented as means $\pm S D, n=4$ in each time point per group. (TIF $532 \mathrm{~kb}$ )
\end{abstract}

Additional file 2: Effects of rh-TSG-6 on neuronal degenerating at $72 \mathrm{~h}$ after subarachnoid hemorrhage (SAH). Representative microphotographs and quantitative analysis of Fluoro-Jade $C$ staining (FJC)-positive neurons in the sham, SAH, vehicle and rh-TSG-6 groups at $72 \mathrm{~h}$ following operation. $n=6$ in each group. Data are expressed as mean \pm SD. ${ }^{* *} P<0.001$. Scale bar $=20 \mu \mathrm{m}$. (TIF $856 \mathrm{~kb}$ )

Additional file 3: Microglial phenotypic characteristics after SAH induction. Photomicrograph of microglia(red) and DAPI(blue) double immunostaining in a coronal section of the whole brain in sham and SAH groups. $n=5$ in each group. Scale bar $=2000 \mu \mathrm{m}$. (TIF $1507 \mathrm{~kb}$ )

\section{Abbreviations}

APC: Allophycocyanin; CNS: Central nervous system; EBI: Early brain injury; FITC: Fluorescein isothiocyanate; FJC: Fluoro-Jade C; GFAP: Glial fibrillary 
acidic protein; Iba1: lonized calcium-binding adapter molecule 1; $\mathrm{ICH}$ : Intracerebral hemorrhage; NeuN: Neuronal nuclear protein; PE: Phycoerythrin; qRT-PCR: Quantitative real-time polymerase chain reaction; SAH: Subarachnoid hemorrhage; SCI: Spinal cord injury; SD: Sprague-Dawley; siRNA: Small interfering RNA; SOCS3: Suppressor of cytokine signaling 3; STAT3: Signal transducer and activator of transcription 3; TSG-6: TNF-a stimulated gene/protein 6

\section{Funding}

This study was supported by the National Natural Science Foundation of China (No. 81271315), the Science and Technology Project Foundation of Guangdong Province (Grant number 2016A020215098), and the Key Project of Clinical Research of Southern Medical University (Grant number LC2016ZD024)

\section{Availability of data and materials}

Information about the experimental methods, animal model, and data used and/or analyzed during the current study available from the corresponding author on reasonable request.

\section{Authors' contributions}

CZD and RL conceived and designed the experiments. RL performed the experiments, drafted the manuscript, and analyzed the data. RL and WCL performed the animal model establishment. JY, YCC, SQG, HYF, XFL, XZ, and $\mathrm{XYH}$ provided the experimental technical support and assisted in completing the study at different stages. CZD reviewed and performed the edition of the paper. All authors checked and approved the final manuscript.

\section{Ethics approval and consent to participate}

All experimental procedures and animal care were approved by the Southern Medical University Ethics Committee and were conducted in accordance with the policy of the National Institutes of Health on the care and use of animals.

\section{Consent for publication}

Not applicable.

\section{Competing interests}

The authors declare that they have no competing interests.

\section{Publisher's Note}

Springer Nature remains neutral with regard to jurisdictional claims in published maps and institutional affiliations.

\section{Received: 10 April 2018 Accepted: 13 August 2018}

Published online: 20 August 2018

\section{References}

1. Macdonald RL, Schweizer TA. Spontaneous subarachnoid haemorrhage. Lancet. 2017;389:655-66.

2. Brown RD, Broderick JP. Unruptured intracranial aneurysms: epidemiology, natural history, management options, and familial screening. The Lancet Neurology. 2014;13:393-404.

3. Korja M, Kaprio J. Controversies in epidemiology of intracranial aneurysms and SAH. Nat Rev Neurol. 2016;12:50-5.

4. de Oliveira Manoel AL, Goffi A, Marotta TR, Schweizer TA, Abrahamson S, Macdonald RL. The critical care management of poor-grade subarachnoid haemorrhage. Crit Care. 2016;20:21.

5. Macdonald RL. Delayed neurological deterioration after subarachnoid haemorrhage. Nat Rev Neurol. 2014;10:44-58.

6. Chen S, Feng H, Sherchan P, Klebe D, Zhao G, Sun X, Zhang J, Tang J, Zhang JH. Controversies and evolving new mechanisms in subarachnoid hemorrhage. Prog Neurobiol. 2014;115:64-91.

7. Young AM, Karri SK, Helmy A, Budohoski KP, Kirollos RW, Bulters DO, Kirkpatrick PJ, Ogilvy CS, Trivedi RA. Pharmacologic Management of Subarachnoid Hemorrhage. World Neurosurg. 2015;84:28-35.

8. Keyrouz SG, Diringer MN. Clinical review: prevention and therapy of vasospasm in subarachnoid hemorrhage. Crit Care. 2007;11:220.

9. van Lieshout JH, Dibué-Adjei M, Cornelius JF, Slotty PJ, Schneider T, Restin T, Boogaarts HD, Steiger HJ, Petridis AK, Kamp MA. An introduction to the pathophysiology of aneurysmal subarachnoid hemorrhage. Neurosurg Rev. 2017. https://doi.org/10.1007/s10143-017-0827-y.
10. Pradilla G, Chaichana KL, Hoang S, Huang J, Tamargo RJ. Inflammation and cerebral vasospasm after subarachnoid hemorrhage. Neurosurg Clin N Am. 2010;21:365-79

11. Chen S, Ma Q, Krafft PR, Hu Q, Rolland W 2nd, Sherchan P, Zhang J, Tang J, Zhang JH. P2X7R/cryopyrin inflammasome axis inhibition reduces neuroinflammation after SAH. Neurobiol Dis. 2013;58:296-307.

12. He Y, Xu L, Li B, Guo ZN, Hu Q, Guo Z, Tang J, Chen Y, Zhang Y, Tang J, Zhang JH. Macrophage-inducible C-type lectin/spleen tyrosine kinase signaling pathway contributes to neuroinflammation after subarachnoid hemorrhage in rats. Stroke. 2015;46:2277-86.

13. Zheng VZ, Wong GKC. Neuroinflammation responses after subarachnoid hemorrhage: a review. J Clin Neurosci. 2017;42:7-11.

14. Ma Y, Wang J, Wang $Y$, Yang GY. The biphasic function of microglia in ischemic stroke. Prog Neurobiol. 2017;157:247-72.

15. Aguzzi A, Barres BA, Bennett ML. Microglia: scapegoat, saboteur, or something else? Science. 2013;339:156-61.

16. Brown GC, Vilalta A. How microglia kill neurons. Brain Res. 2015;1628: 288-97.

17. ladecola C, Anrather J. The immunology of stroke: from mechanisms to translation. Nat Med. 2011;17:796-808

18. Franco R, Fernandez-Suarez D. Alternatively activated microglia and macrophages in the central nervous system. Prog Neurobiol. 2015;131:65-86.

19. Bouhlel MA, Derudas B, Rigamonti E, Dievart R, Brozek J, Haulon S, Zawadzki C, Jude B, Torpier G, Marx N, et al. PPARgamma activation primes human monocytes into alternative M2 macrophages with anti-inflammatory properties. Cell Metab. 2007;6:137-43.

20. Tentillier N, Etzerodt A, Olesen MN, Rizalar FS, Jacobsen J, Bender D, Moestrup SK, Romero-Ramos M. Anti-inflammatory modulation of microglia via CD163-targeted glucocorticoids protects dopaminergic neurons in the 6-OHDA Parkinson's disease model. J Neurosci. 2016;36:9375-90.

21. Francos-Quijorna I, Amo-Aparicio J, Martinez-Muriana A, Lopez-Vales R. IL-4 drives microglia and macrophages toward a phenotype conducive for tissue repair and functional recovery after spinal cord injury. Glia. 2016; 64:2079-92

22. Shi H, Zheng K, Su Z, Su H, Zhong M, He X, Zhou C, Chen H, Xiong Q, Zhang $Y$. Sinomenine enhances microglia M2 polarization and attenuates inflammatory injury in intracerebral hemorrhage. J Neuroimmunol. 2016;299:28-34.

23. Yang $Y$, Liu $H$, Zhang $H$, Ye Q, Wang J, Yang B, Mao L, Zhu W, Leak RK, Xiao $B$, et al. ST2/IL-33-dependent microglial response limits acute ischemic brain injury. J Neurosci. 2017;37:4692-704.

24. Xu H, Wang Z, Li J, Wu H, Peng Y, Fan L, Chen J, Gu C, Yan F, Wang L, Chen $G$. The polarization states of microglia in TBI: a new paradigm for pharmacological intervention. Neural Plast. 2017;2017:5405104.

25. Milner CM, Day AJ. TSG-6: a multifunctional protein associated with inflammation J Cell Sci. 2003:116:1863-73.

26. Day AJ, Milner CM. TSG-6: a multifunctional protein with anti-inflammatory and tissue-protective properties. Matrix Biol. 2018. https://doi.org/10.1016/j. matbio.2018.01.011.

27. Chen M, Li X, Zhang X, He X, Lai L, Liu Y, Zhu G, Li W, Li H, Fang Q, et al. The inhibitory effect of mesenchymal stem cell on blood-brain barrier disruption following intracerebral hemorrhage in rats: contribution of TSG-6. J Neuroinflammation. 2015;12:61.

28. Zhang $R$, Liu Y, Yan $K$, Chen L, Chen XR, Li P, Chen FF, Jiang XD. Antiinflammatory and immunomodulatory mechanisms of mesenchymal stem cell transplantation in experimental traumatic brain injury. J Neuroinflammation. 2013;10:106.

29. Schwartz AY, Masago A, Sehba FA, Bederson JB. Experimental models of subarachnoid hemorrhage in the rat: a refinement of the endovascular filament model. J Neurosci Methods. 2000;96:161-7.

30. Sugawara $T$, Ayer $R$, Jadhav $V$, Zhang $J H$. A new grading system evaluating bleeding scale in filament perforation subarachnoid hemorrhage rat model. J Neurosci Methods. 2008:167:327-34.

31. Zeng J, Chen Y, Ding R, Feng L, Fu Z, Yang S, Deng X, Xie Z, Zheng S. Isoliquiritigenin alleviates early brain injury after experimental intracerebral hemorrhage via suppressing ROS- and/or NF-kappaB-mediated NLRP3 inflammasome activation by promoting Nrf2 antioxidant pathway. J Neuroinflammation. 2017;14:119.

32. Frank MG, Wieseler-Frank JL, Watkins LR, Maier SF. Rapid isolation of highly enriched and quiescent microglia from adult rat hippocampus: 
immunophenotypic and functional characteristics. J Neurosci Methods. 2006;151:121-30.

33. Chang CF, Wan J, Li Q, Renfroe SC, Heller NM, Wang J. Alternative activation-skewed microglia/macrophages promote hematoma resolution in experimental intracerebral hemorrhage. Neurobiol Dis. 2017;103:54-69.

34. Watanabe J, Shetty AK, Hattiangady B, Kim DK, Foraker JE, Nishida H, Prockop DJ. Administration of TSG-6 improves memory after traumatic brain injury in mice. Neurobiol Dis. 2013;59:86-99.

35. Faustino JV, Wang X, Johnson CE, Klibanov A, Derugin N, Wendland MF, Vexler ZS. Microglial cells contribute to endogenous brain defenses after acute neonatal focal stroke. J Neurosci. 2011;31:12992-3001.

36. Fernandez-Lopez D, Faustino J, Klibanov AL, Derugin N, Blanchard E, Simon F, Leib SL, Vexler ZS. Microglial cells prevent hemorrhage in neonatal focal arterial stroke. J Neurosci. 2016;36:2881-93.

37. Coulson-Thomas VJ, Lauer ME, Soleman S, Zhao C, Hascall VC, Day AJ, Fawcett JW. Tumor necrosis factor-stimulated Gene-6 (TSG-6) is constitutively expressed in adult central nervous system (CNS) and associated with astrocytemediated glial scar formation following spinal cord injury. J Biol Chem. 2016; 291:19939-52.

38. Li T, Pang S, Yu Y, Wu X, Guo J, Zhang S. Proliferation of parenchymal microglia is the main source of microgliosis after ischaemic stroke. Brain 2013;136:3578-88

39. Pan J, Jin JL, Ge HM, Yin KL, Chen X, Han LJ, Chen Y, Qian L, Li XX, Xu Y. Malibatol A regulates microglia M1/M2 polarization in experimental stroke in a PPARgamma-dependent manner. J Neuroinflammation. 2015;12:51.

40. Wisniewski HG, Colon E, Liublinska V, Karia RJ, Stabler TV, Attur M, Abramson SB, Band PA, Kraus VB. TSG-6 activity as a novel biomarker of progression in knee osteoarthritis. Osteoarthr Cartil. 2014;22:235-41.

41. Ransohoff RM. A polarizing question: do M1 and M2 microglia exist? Nat Neurosci. 2016;19:987-91.

42. He Y, Ma X, Li D, Hao J. Thiamet $G$ mediates neuroprotection in experimental stroke by modulating microglia/macrophage polarization and inhibiting NFkappaB p65 signaling. J Cereb Blood Flow Metab. 2017;37:2938-51.

43. Jang E, Lee S, Kim JH, Kim JH, Seo JW, Lee WH, Mori K, Nakao K, Suk K. Secreted protein lipocalin-2 promotes microglial M1 polarization. FASEB J. 2013;27:1176-90.

44. Xie Y, Guo H, Wang L, Xu L, Zhang X, Yu L, Liu Q, Li Y, Zhao N, Zhao N, et al. Human albumin attenuates excessive innate immunity via inhibition of microglial Mincle/Syk signaling in subarachnoid hemorrhage. Brain Behav Immun. 2017:60:346-60.

45. Schilling M, Besselmann M, Leonhard C, Mueller M, Ringelstein EB, Kiefer R. Microglial activation precedes and predominates over macrophage infiltration in transient focal cerebral ischemia: a study in green fluorescent protein transgenic bone marrow chimeric mice. Exp Neurol. 2003;183:25-33.

46. d'Avila JC, Lam TI, Bingham D, Shi J, Won SJ, Kauppinen TM, Massa S, Liu J, Swanson RA. Microglial activation induced by brain trauma is suppressed by post-injury treatment with a PARP inhibitor. J Neuroinflammation. 2012;9:31.

47. Lucke-Wold BP, Logsdon AF, Manoranjan B, Turner RC, McConnell E, Vates $\mathrm{GE}$, Huber JD, Rosen CL, Simard JM. Aneurysmal subarachnoid hemorrhage and neuroinflammation: a comprehensive review. Int J Mol Sci. 2016;17:497.

48. Liang Z, Wu G, Fan C, Xu J, Jiang S, Yan X, Di S, Ma Z, Hu W, Yang Y. The emerging role of signal transducer and activator of transcription 3 in cerebral ischemic and hemorrhagic stroke. Prog Neurobiol. 2016;137:1-16.

49. Satriotomo I, Bowen KK, Vemuganti R. JAK2 and STAT3 activation contributes to neuronal damage following transient focal cerebral ischemia. J Neurochem. 2006;98:1353-68.

50. Sehara Y, Sawicka K, Hwang JY, Latuszek-Barrantes A, Etgen AM, Zukin RS. Survivin is a transcriptional target of STAT3 critical to estradiol neuroprotection in global ischemia. J Neurosci. 2013;33:12364-74.

51. Oliva AA Jr, Kang Y, Sanchez-Molano J, Furones C, Atkins CM. STAT3 signaling after traumatic brain injury. J Neurochem. 2012;120:710-20.

52. Nakamura R, Sene A, Santeford A, Gdoura A, Kubota S, Zapata N, Apte RS, IL10-driven STAT3 signalling in senescent macrophages promotes pathological eye angiogenesis. Nat Commun. 2015;6:7847.

53. O'Shea JJ, Murray PJ. Cytokine signaling modules in inflammatory responses. Immunity. 2008;28:477-87.

54. Croker BA, Krebs DL, Zhang JG, Wormald S, Willson TA, Stanley EG, Robb L, Greenhalgh CJ, Forster I, Clausen BE, et al. SOCS3 negatively regulates IL-6 signaling in vivo. Nat Immunol. 2003;4:540-5.
55. Qin H, Yeh WI, De Sarno P, Holdbrooks AT, Liu Y, Muldowney MT, Reynolds SL, Yanagisawa LL, Fox TH 3rd, Park K, et al. Signal transducer and activator of transcription-3/suppressor of cytokine signaling-3 (STAT3/SOCS3) axis in myeloid cells regulates neuroinflammation. Proc Natl Acad Sci U S A. 2012; 109:5004-9.

56. Chen S, Dong Z, Cheng M, Zhao Y, Wang M, Sai N, Wang X, Liu H, Huang G, Zhang X. Homocysteine exaggerates microglia activation and neuroinflammation through microglia localized STAT3 overactivation following ischemic stroke. J Neuroinflammation. 2017;14:187.

57. Zhai Q, Li F, Chen X, Jia J, Sun S, Zhou D, Ma L, Jiang T, Bai F, Xiong L, Wang $\mathrm{Q}$. Triggering receptor expressed on myeloid cells 2, a novel regulator of immunocyte phenotypes, confers neuroprotection by relieving neuroinflammation. Anesthesiology. 2017;127:98-110.

58. Mittal M, Tiruppathi C, Nepal S, Zhao YY, Grzych D, Soni D, Prockop DJ, Malik AB. TNFalpha-stimulated gene-6 (TSG6) activates macrophage phenotype transition to prevent inflammatory lung injury. Proc Natl Acad Sci U S A. 2016;113:E8151-e8158.

\section{Ready to submit your research? Choose BMC and benefit from:}

- fast, convenient online submission

- thorough peer review by experienced researchers in your field

- rapid publication on acceptance

- support for research data, including large and complex data types

- gold Open Access which fosters wider collaboration and increased citations

- maximum visibility for your research: over $100 \mathrm{M}$ website views per year

At BMC, research is always in progress.

Learn more biomedcentral.com/submissions 